\title{
Multiband nonthermal radiative properties of pulsar wind nebulae
}

\author{
Bo-Tao Zhu, Li Zhang, and Jun Fang
}

\begin{abstract}
Department of Astronomy, Key Laboratory of Astroparticle Physics of Yunnan Province, Yunnan University, 650091 Kunming, PR China

e-mail: [lizhang; fangjun]@ynu.edu.cn
\end{abstract}

Received 14 June 2016 / Accepted 11 July 2017

\begin{abstract}
Aims. The nonthermal radiative properties of 18 pulsar wind nebulae (PWNe) are studied in the 1D leptonic model.

Methods. The dynamical and radiative evolution of a PWN in a nonradiative supernova remnant are self-consistently investigated in this model. The leptons (electrons/positrons) are injected with a broken power-law form, and nonthermal emission from a PWN is mainly produced by time-dependent relativistic leptons through synchrotron radiation and inverse Compton process.

Results. Observed spectral energy distributions (SEDs) of all 18 PWNe are reproduced well, where the indexes of low-energy electron components lie in the range of 1.0-1.8 and those of high-energy electron components in the range of 2.1-3.1. Our results show that $F_{\mathrm{X}} / F_{\gamma}>10$ for young PWNe; $1<F_{\mathrm{X}} / F_{\gamma} \leq 10$ for evolved PWNe, except for G292.0+1.8; and $F_{\mathrm{X}} / F_{\gamma} \leq 1$ for mature/old PWNe, except for CTA 1. Moreover, most PWNe are particle-dominated. Statistical analysis for the sample of 14 PWNe further indicate that (1) not all pulsar parameters have correlations with electron injection parameters, but electron maximum energy and PWN magnetic field correlate with the magnetic field at the light cylinder, the potential difference at the polar cap, and the spindown power; (2) the spin-down power positively correlates with radio, X-ray, bolometric, and synchrotron luminosities, but does not correlate with gamma-ray luminosity; (3) the spin-down power positively correlates with radio, X-ray, and $\gamma$-band surface brightness; and (4) the PWN radius and the PWN age negatively correlate with X-ray luminosity, the ratio of X-ray to gamma-ray luminosities, and the synchrotron luminosity.
\end{abstract}

Key words. pulsars: general - stars: winds, outflows - acceleration of particles - radiation mechanisms: non-thermal

\section{Introduction}

A pulsar wind nebula (PWN) is formed when the wind of a pulsar interacts with the ambient medium, either the supernova ejecta or the interstellar medium (e.g., Rees \& Gunn 1974; Kennel \& Coroniti 1984; Chevalier 2004; Gaensler \& Slane 2006; Bucciantini 2008). It is generally believed that a PWN is mainly composed of a relativistic nonthermal lepton (electron/positron) plasma and magnetic field and can emit nonthermal photons from radio to $\gamma$-rays via synchrotron radiation and the inverse Compton (IC) process. Observations have shown that some PWNe, such as the Crab nebula, Kes 75, MSH 15-52, can emit nonthermal emission from radio to very high-energy (VHE) $\gamma$-ray bands (e.g., Bühler \& Blandford 2014; Reynolds et al. 2017). Currently, about $37 \mathrm{TeV}$ PWNe are firmly identified $^{1}$. More recently, the observations and relevant physical analysis of $19 \mathrm{TeV}$ PWNe have been presented in Abdalla et al. (2017), where $14 \mathrm{TeV}$ PWNe are firmly identified as PWNe by HESS observations. These observations provide an essential reason for studying PWNe.

Various models have been proposed to explain the nonthermal properties of PWNe and a brief review of current models and differences can be found in Torres et al. (2014). Here we focus on the one-dimensional (1D) model of the dynamical and radiative evolution of a PWN in a nonradiative SNR. Here we focus on the 1D model of the dynamical and radiative evolution of a PWN in a nonradiative supernova remnant (SNR) presented in Gelfand et al. (2009). In the frame of the model, the radiative properties during different phases of the PWN evolution

\footnotetext{
1 http://tevcat.uchicago.edu/
}

are investigated with a single power-law injection spectrum for the electrons/positrons (Gelfand et al. 2009), with the relativistic Maxwellian and a high-energy power-law tail injection spectrum for the electrons/positrons (Fang \& Zhang 2010a), and with two possible forms of injected electron spectra: a broken power law and the sum of a power law at low energy, and a relativistic Maxwellian plus a high-energy power-law tail (Zhu et al. 2015). It has been shown that the broken power-law form for the electron injection is required when the model applies to the Crab nebula (Zhu et al. 2015). Recently, a model for describing PWN radiative properties during the dynamical evolutions of the PWN and the SNR is presented in Martin et al. (2016), the radius of the PWN during the free expansion phase and compression in this model is calculated through solving the equations given by Chevalier (2005), where the prescription is similar to that in Gelfand et al. (2009). The model is applied to a TeV PWN CTA 1 (Martin et al. 2016). We note that there is a kind of model in which a broken power-law injection spectrum for the electrons/positrons is assumed but the dynamics beyond reverberation is not included (e.g., Zhang et al. 2008; Bucciantini et al. 2011; Tanaka \& Takahara 2011).

To investigate nonthermal radiative properties of each PWN and statistical features of PWNe, observed multiwaveband data for each PWN are required. However, not all PWNe are observed at different bands, due to observation limits, so $18 \mathrm{PWNe}$ from known PWNe are selected here according to the following criteria: (1) the period and period derivative of central pulsar in each PWN are known, and (2) nonthermal emission at radio, X-ray, and $\mathrm{TeV}$ bands are detected. In this selection, $\mathrm{N} 158 \mathrm{~A}$ is not detected at TeV band, G310.6-1.6 is detected with an upper limit at $\mathrm{TeV}$ band, G292.0+1.8 is detected with upper limits at $\mathrm{GeV}$ 
band. These three PWNe are included in the sample because of their energetic pulsars. These PWNe are divided into three groups based on the ages of PWNe that are adopted in our calculations (see Sect. 3). The nonthermal radiative property for each PWN is studied in the leptonic model with a broken power-law injection for the electrons/positrons, and the correlation features of all $18 \mathrm{PWNe}$ are presented.

The structure of this article is as follows. In Sect. 2 we briefly review the model. In Sect. 3 we apply the model to 18 PWNe, and calculate the spectral energy distibution (SED) of each PWN. In Sect. 4 we study correlation properties of the PWNe, and we give our conclusions and discussions in Sect. 5.

\section{Model description}

For completeness, we briefly review the model for dynamical and radiative evolution of a PWN inside a SNR given in Zhu et al. (2015), which is closely based on that developed by Gelfand et al. (2009; see also Fang \& Zhang 2010a). In this model, the large-scale evolution of a composite SNR depends on the mechanical energy $E_{\mathrm{Sn}}$ of the explosion, the density $n_{\mathrm{H}}$ of the ambient medium, the mass $M_{\mathrm{ej}}$ of the supernova ejecta, and the spin-down power $L(t)$ of the pulsar. For a given pulsar with a period $P$, a period derivative $\dot{P}$, a braking index $n$, and an initial spin-down power $L_{0}$, the spin-down power $L(t)$ at time $t$ is given by (Gaensler \& Slane 2006)

$L(t)=L_{0}\left(1+\frac{t}{\tau_{0}}\right)^{-(n+1) /(n-1)}$,

where $\tau_{0}=\left(2 \tau_{\mathrm{c}}\right) /(n-1)-T_{\text {age }}$ is the initial spin-down timescale of the pulsar, $\tau_{\mathrm{c}}=P / 2 \dot{P}$ is the characteristic age of the pulsar, and $T_{\text {age }}$ is the age of the PWN. Since $L(t)$ can be estimated by $L(t)=4 \pi^{2} I \dot{P} / P^{3}$, where $I$ is the pulsar moment of inertia (here $I=10^{45} \mathrm{~g} \mathrm{~cm}^{2}$ is used), the evolution of the spin-down power can be determined (i.e., $L_{0}$ and $\tau_{0}$ can be estimated) if $T_{\text {age }}$ is known. Therefore, the main parameters of a given pulsar are $P$, $\dot{P}$, and $n$. At present, the vaules of the braking index $n$ for some pulsars have been measured, and we use the measured values if available, otherwise we assume $n=3$. We call these parameters the pulsar and ejecta parameters.

The evolution of isotropic electron distribution $N(E, t)$ in the PWN is calculated by using

$\frac{\partial N(E, t)}{\partial t}=\frac{\partial}{\partial E}[\dot{E} N(E, t)]+\frac{N(E, t)}{\tau(E, t)}+Q(E, t)$,

where $\dot{E}$ is the energy-loss rate of the particles with an energy $E$ which includes the contributions of synchrotron radiation, inverse Compton scattering, and adiabatic losses and $\tau(E, t)$ is the escape time (for the details of their processes, see Zhang et al. 2008). The last term on the right-hand side in Eq. (2), $Q(E, t)$, is a source term, i.e., the injected electron number per unit energy per unit time, and is assumed to be a broken power-law form

$Q(E, t)=Q_{0}(t) \begin{cases}\left(\frac{E}{E_{\mathrm{b}}}\right)^{-\alpha_{1}} & \text { for } E \leq E_{\mathrm{b}}, \\ \left(\frac{E}{E_{\mathrm{b}}}\right)^{-\alpha_{2}} & \text { for } E_{\mathrm{b}} \leq E<E_{\mathrm{max}},\end{cases}$

where $Q_{0}(t)$ is a time-dependent parameter, $E_{\mathrm{b}}$ is the break energy, $\alpha_{1}<2$ and $\alpha_{2}>2$ are respectively the spectral indexes of the injection rate at $E \leq E_{\mathrm{b}}$ and $E>E_{\mathrm{b}}$, and $E_{\max }$ is the maximum energy of the electrons.

Here the spin-down power of the pulsar is assumed to be distributed between electrons $\left(\dot{E}_{\mathrm{e}}(t)=\eta_{\mathrm{e}} L(t)\right)$ and magnetic fields
$\left(\dot{E}_{\mathrm{B}}=\eta_{\mathrm{B}} L(t)\right)\left[\eta_{\mathrm{e}}+\eta_{\mathrm{B}}=1\right]$ (Gelfand et al. 2009), then $Q_{0}(t)$ can be estimated from $\left(1-\eta_{\mathrm{B}}\right) L(t)=\int_{0}^{E_{\max }} Q(E, t) E \mathrm{~d} E$, which gives

$$
Q_{0}(t)= \begin{cases}\frac{\left(1-\eta_{\mathrm{B}}\right) L(t)}{E_{\mathrm{b}}{ }^{2}} \frac{\left(2-\alpha_{1}\right)\left(2-\alpha_{2}\right)}{\alpha_{1}-\alpha_{2}} & \alpha_{2}>2 \\ \frac{\left(1-\eta_{\mathrm{B}}\right) L(t)}{E_{b}{ }^{2}}\left[\frac{1}{2-\alpha_{1}}+\log \left(\frac{E_{\max }}{E_{\mathrm{b}}}\right)\right] & \alpha_{2}=2 .\end{cases}
$$

On the other hand, the maximum energy of the electrons can be estimated by the condition that the Larmor radius of the electrons inside the PWN is smaller than the termination shock radius of the PWNe by the containment factor $\epsilon<1$, which is given by

$E_{\max } \approx 3 \epsilon e \sqrt{\frac{\eta_{\mathrm{B}} L(t)}{c}}$,

where $e$ is the electron charge (e.g., Zhu et al. 2015). We note that $E_{\max }$ can also be estimated by the balance between the synchrotron loses and acceleration, which gives $E_{\max }^{\mathrm{syn}} \approx$ $\left[3\left(m_{\mathrm{e}} c^{2}\right)^{2} / 4 e\right] \sqrt{\pi / e B_{\text {pwn }}}$. For the parameters of PWNe used here (see Tables 1-3), $E_{\max }<E_{\max }^{\text {syn }}$ is always satisfied. Hence, the injection parameters involving electron injection are $E_{\mathrm{b}}, \alpha_{1}, \alpha_{2}$, $\eta_{\mathrm{B}}$, and $\epsilon$.

In such a model, the dynamical and radiative properties for a given PWN can be self-consistently studied Gelfand et al. (2009; see also Fang \& Zhang 2010a; and Zhu et al. 2015). On the one hand, the time evolutions of the SNR radius $R_{\mathrm{snr}}(t)$, reverse shock radius $R_{\mathrm{rs}}(t)$, PWN radius $R_{\mathrm{pwn}}(t)$, the position of the neutron star $R_{\mathrm{psr}}(t)$, the termination shock radius $R_{\mathrm{ts}}(t)$, and the magnetic field $B_{\mathrm{pwn}}(t)$ of the PWN can be calculated. On the other hand, the time evolutions of electron spectrum and the spectral energy distribution (SED) of nonthermal photons can be obtained. It should be pointed out that different electron injection forms and electron energy losses will lead to the change in dynamical features of the PWN. In this paper, the broken power-law injection of the electrons (see Eq. (3)) and escape term of the electrons (see the second term on the left-hand side of Eq. (2)) are considered, so the dynamical features for a given PWN will be different from those given in Gelfand et al. (2009), who assumed a single power-law injection without electron escape (e.g., Crab nebulae, see Zhu et al. 2015). We note that both $R_{\text {pwn }}(t)$ and $B_{\text {pwn }}(t)$ play important roles in the evolution of the energy losses and the SED for a given PWN. Therefore, in our calculations the allowed ranges of $R_{\text {pwn }}(t)$ are limited by the values given in Abdalla et al. (2017; see their Tables 1 and 3).

In our calculations, nonthermal photons are produced through synchrotron radiation and inverse Compton (IC) scattering of relativistic electrons (e.g., Zhu et al. 2015). For the synchrotron radiation, the emissivity given in Zhang et al. (2008) is used, which includes the effect of electron pitch angle. The magnetic field $B_{\text {pwn }}$ of a PWN evolves with time and can be estimated by Eq. (A.6) in Appendix A. We note that Torres et al. (2014) did not consider the effect of electron pitch angle (see also Tanaka \& Takahara 2010; Martin et al. 2012), which will result in parameter differences between our model and the model in Torres et al. (2014) for a given PWN.

For IC scattering, soft photon fields consist of four components: the cosmic microwave background (CMB) in our calculations, the galactic far-infrared (FIR) background, the near-infrared (NIR) and optical photon field due to the stars, and synchrotron radiation. The energy density and temperature of the CMB photons are $U_{\mathrm{CMB}}=0.25 \mathrm{eV} \mathrm{cm}^{-3}$ and $T_{\mathrm{CMB}}=2.7 \mathrm{~K}$, and the energy densities and temperatures of FIR ( $U_{\mathrm{FIR}}$ and $\left.T_{\mathrm{FIR}}\right)$ 
Table 1. Parameters for young PWNe.

\begin{tabular}{|c|c|c|c|c|c|c|}
\hline PWN & N 158A & G21.5-0.9 & Crab nebula & Kes 75 & G310.6-1.6 & $3 C 58$ \\
\hline \multicolumn{7}{|c|}{ Pulsar and ejecta parameters } \\
\hline$P(\mathrm{~ms})$ & 50.5 & 61.8 & 33.4 & 324.0 & 31.18 & 65.7 \\
\hline$\dot{P}\left(\mathrm{~s} \mathrm{~s}^{-1}\right)$ & $4.79 \times 10^{-13}$ & $2.02 \times 10^{-13}$ & $4.23 \times 10^{-13}$ & $7.08 \times 10^{-12}$ & $3.89 \times 10^{-14}$ & $1.93 \times 10^{-13}$ \\
\hline$n$ & 2.129 & 3.0 & 2.509 & 2.19 & 3.0 & 3.0 \\
\hline$\tau_{\mathrm{c}}(\mathrm{yr})$ & 1671 & 4850 & 1296 & 726 & 12708 & 5397 \\
\hline$L(t)\left(\mathrm{erg} \mathrm{s}^{-1}\right)$ & $1.47 \times 10^{38}$ & $3.38 \times 10^{37}$ & $4.53 \times 10^{38}$ & $8.21 \times 10^{36}$ & $5.06 \times 10^{37}$ & $2.68 \times 10^{37}$ \\
\hline$T_{\text {age }}(\mathrm{yr})$ & 760 & 900 & 940 & 1000 & 1500 & 2400 \\
\hline$L_{0}\left(\mathrm{erg} \mathrm{s}^{-1}\right)$ & $3.34 \times 10^{38}$ & $5.09 \times 10^{37}$ & $3.1 \times 10^{39}$ & $8.11 \times 10^{38}$ & $6.51 \times 10^{37}$ & $8.69 \times 10^{37}$ \\
\hline$\tau_{0}(\mathrm{yr})$ & 2201 & 3950 & 730 & 220 & 11208 & 2997 \\
\hline$M_{\mathrm{ej}}\left(M_{\odot}\right)$ & 23.0 & 8.0 & 9.5 & 10.0 & 13.0 & 8.0 \\
\hline$E_{\mathrm{SN}}\left(10^{51} \mathrm{erg}\right)$ & 2.0 & 1.0 & 1.0 & 1.0 & 1.0 & 1.0 \\
\hline$n_{\mathrm{H}}\left(\mathrm{cm}^{3}\right)$ & 0.01 & 0.1 & 0.1 & 0.1 & 0.01 & 0.01 \\
\hline$d(\mathrm{kpc})$ & 49 & 4.1 & 2.0 & 5.8 & 7.0 & 2.0 \\
\hline \multicolumn{7}{|c|}{ Soft photon parameters } \\
\hline$T_{\text {FIR }}(\mathrm{K})$ & 88.0 & 35.0 & 70.0 & 30.0 & 40.0 & 40.0 \\
\hline$U_{\mathrm{FIR}}\left(\mathrm{eV} \mathrm{cm}^{-3}\right)$ & 1.0 & 1.4 & 0.5 & 1.2 & 0.3 & 0.3 \\
\hline$T_{\mathrm{NIR}}(\mathrm{K})$ & 5000.0 & 3500.0 & 5000.0 & 3000.0 & 4000.0 & 4000.0 \\
\hline$U_{\mathrm{NIR}}\left(\mathrm{eV} \mathrm{cm}^{-} 3\right)$ & 1.0 & 5.0 & 1.0 & 2.2 & 0.3 & 0.3 \\
\hline \multicolumn{7}{|c|}{ Injection parameters } \\
\hline$E_{\mathrm{b}}\left(10^{5} \mathrm{MeV}\right)$ & 150.00 & 1.52 & 6.26 & 2.13 & 6.50 & 0.61 \\
\hline$\alpha_{1}$ & 1.75 & 1.00 & 1.59 & 1.55 & 1.06 & 1.03 \\
\hline$\alpha_{2}$ & $2.31 \pm 0.19$ & $2.63 \pm 0.01$ & $2.42 \pm 0.01$ & $2.09 \pm 0.03$ & $2.71 \pm 0.06$ & $3.07 \pm 0.02$ \\
\hline$\eta_{\mathrm{B}}$ & $0.0014 \pm 0.0004$ & $0.046 \pm 0.006$ & $0.040 \pm 0.001$ & $0.0019 \pm 0.0004$ & $0.0017 \pm 0.0002$ & $0.061 \pm 0.008$ \\
\hline$\epsilon$ & 0.30 & $0.32 \pm 0.04$ & $0.14 \pm 0.01$ & $0.58 \pm 0.23$ & 0.12 & $0.064 \pm 0.045$ \\
\hline Chi-squared $\chi^{2}$ & 0.20 & 1.35 & 5.73 & 2.08 & 4.88 & 1.91 \\
\hline \multicolumn{7}{|c|}{ Derived parameters } \\
\hline$E_{\max }\left(10^{8} \mathrm{MeV}\right)$ & $7.06_{-1.09}^{+0.95}$ & $20.78_{-3.83}^{+4.07}$ & $30.91_{-2.57}^{+2.62}$ & $3.76_{-1.74}^{+2.02}$ & $1.83_{-0.12}^{+0.10}$ & $4.25_{-3.07}^{+3.44}$ \\
\hline$B_{\mathrm{pwn}}(\mu \mathrm{G})$ & $45.22_{-9.65}^{+14.81}$ & $84.35_{-7.52}^{+7.13}$ & $116.34_{-1.02}^{+0.98}$ & $14.18_{-2.69}^{+3.33}$ & $13.16_{-0.91}^{+0.90}$ & $23.92_{-1.81}^{+1.71}$ \\
\hline$R_{\mathrm{pwn}}(\mathrm{pc})$ & 0.68 & 0.86 & 1.95 & 0.99 & 1.32 & 3.05 \\
\hline$R_{\mathrm{rs}}(\mathrm{pc})$ & 4.64 & 4.04 & 4.00 & 4.12 & 6.58 & 10.03 \\
\hline$R_{\mathrm{snr}}(\mathrm{pc})$ & 5.52 & 4.81 & 4.76 & 4.91 & 7.83 & 11.93 \\
\hline$R_{\mathrm{ts}}(\mathrm{pc})$ & 0.05 & 0.06 & 0.20 & 0.05 & 0.09 & 0.23 \\
\hline
\end{tabular}

and NIR ( $U_{\text {NIR }}$ and $T_{\text {NIR }}$ ) can be different for PWNe. We refer to these parameters as soft photon parameters. The emissivity of IC scattering used here is given by Zhang et al. (2008).

To fit the observed spectral energy distribution (SED) for each PWN, pulsar and ejecta parameters and soft photon parameters are fixed, and injection parameters are considered as fitting parameters (see Tables 1-3). The Levenberg-Marquardt (LM) method of the $\chi^{2}$ minimization fitting procedure is used to find the best-fitting values of injection parameters and their uncertainties. However, because of the lack of data (from radio to optical) for most PWNe, the best-fitting values and their uncertainties of $\alpha_{1}$ and $E_{\mathrm{b}}$ cannot be easily obtained. Therefore, at first, visual fitting is used to determine the values of $\alpha_{1}$ and $E_{\mathrm{b}}$, and then the LM method is used to obtain the values of $\alpha_{2}, \eta_{\mathrm{B}}$,

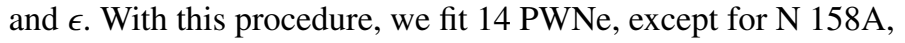
G310.6-1.6, G292.0+1.8, and HESS J1303-631. Since the observed data at GeV-TeV and from radio to X-ray band are upper limits for these four PWNe, the LM method can only determine the values of $\alpha_{2}$ and $\eta_{\mathrm{B}}$ for $\mathrm{N} 158 \mathrm{~A}, \mathrm{G} 310.6-1.6$, and G292.0+1.8, and $\alpha_{2}$ for HESS J1303-631; other injection parameters are estimated via visual fitting. These values are shown in Tables 1-3.
In addition to the above parameters, six derived parameters $\left(E_{\mathrm{max}}, B_{\mathrm{pwn}}, R_{\mathrm{pwn}}, R_{\mathrm{rs}}, R_{\mathrm{snr}}\right.$, and $\left.R_{\mathrm{ts}}\right)$ are also given (see Tables 1-3). We note that $E_{\max }$ and $B_{\text {pwn }}$, which relate to $\epsilon$ and $\eta_{\mathrm{B}}$, are calculated. Because the slight difference in the injection spectrum has little effect on the dynamical properties of PWNe, the uncertainties of $R_{\mathrm{pwn}}, R_{\mathrm{rs}}, R_{\mathrm{snr}}$, and $R_{\mathrm{ts}}$ are not calculated here.

\section{Applications to individual PWNe}

The model described in Sect. 2 is now applied to explain the observed SEDs of nonthermal photons for $18 \mathrm{PWNe}$. The detailed descriptions of observed and derived properties of each PWN are summarized in Appendix B. The PWN sample is divided into three groups based on possible ages $T_{\text {age }}$ of PWNe. The first group consists of the PWNe with $T_{\text {age }} \leq 2400 \mathrm{yr}$ (we call them young PWNe), the second group with $2400<T_{\text {age }}<5000 \mathrm{yr}$ (called evolved PWNe), and the third group with $T_{\text {age }} \geq 5000 \mathrm{yr}$ (called mature/old PWNe). Although the division of the three groups is arbitrary, it is convenient for us to study the properties of PWNe that lie in different age ranges. 

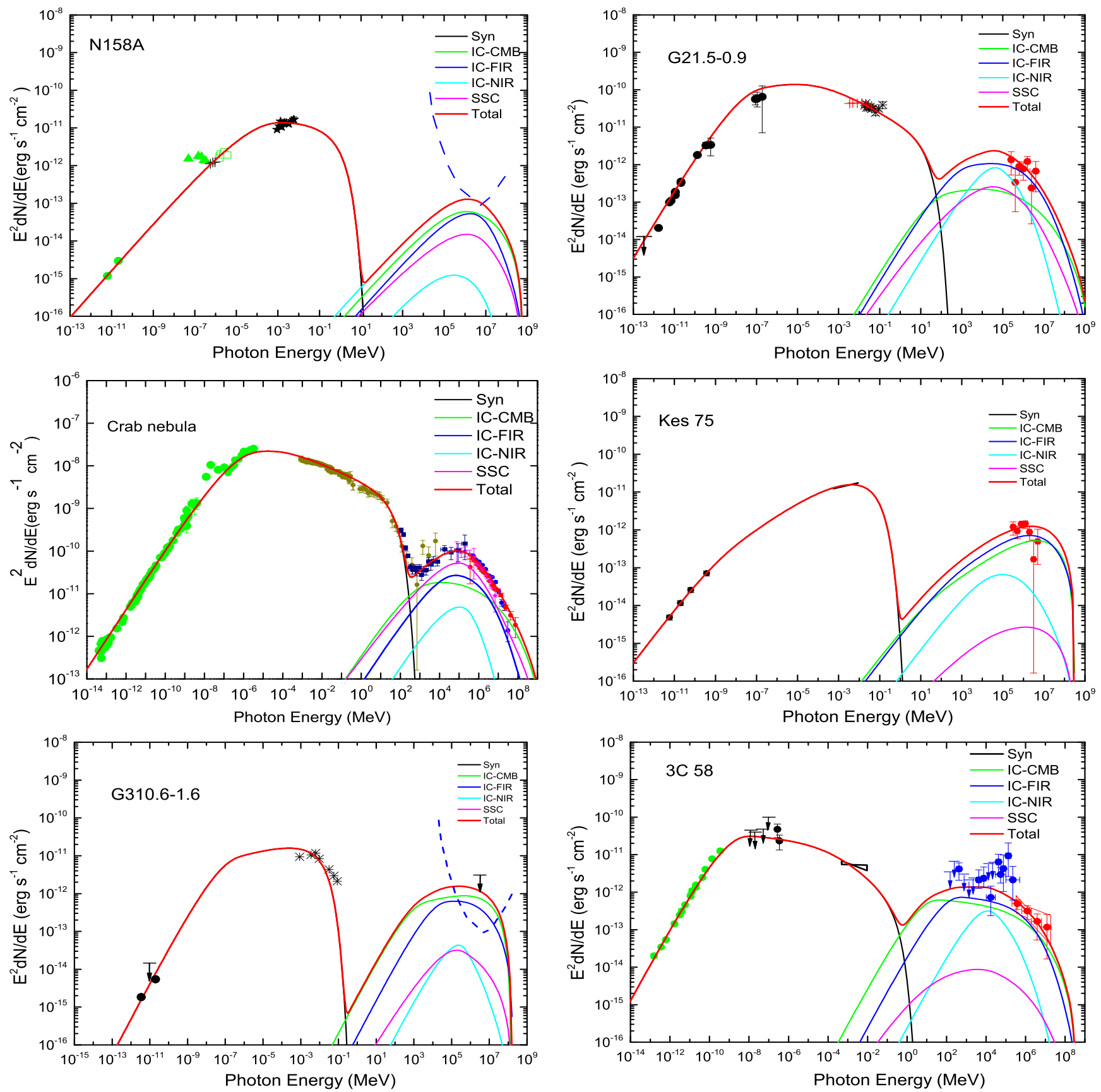

Fig. 1. Comparisons of predicted SEDs and observed data for young PWNe (from upper left panel for N 158A to bottom right panel for 3C 58). In each panel, the black line represents synchrotron SED; the magenta, blue, green, and cyan lines represent the SEDs of inverse Compton scatterings with the synchrotron photons, IR, CMB, and starlight, respectively; and the total SED is shown by the red line. See text for the descriptions of the observed data; the relevant parameters are listed in Table 1.

Some of the 18 PWNe have been already investigated (e.g., Fang \& Zhang 2010a; Tanaka \& Takahara 2011; Torres et al. 2014; Martin et al. 2014; Zhu et al. 2015; Martin et al. 2016). Two important quantities that influence the electron energy loss and photon SED of a PWN are $R_{\mathrm{pwn}}(t)$ and $B_{\mathrm{pwn}}(t)$. In Torres et al. (2014), $R_{\mathrm{pwn}}(t) \propto t^{6 / 5}$ is calculated during the SNR's free expansion phase (van der Swaluw et al. 2001, 2003) and $B_{\text {pwn }}(t)$ is estimated with a method similar to that used in Gelfand et al. (2009). In Tanaka \& Takahara (2011), $B_{\text {pwn }}(t)$ is calculated by using the magnetic field energy conservation, i.e., $(4 \pi / 3) R_{\mathrm{pwn}}^{3}(t) B_{\mathrm{pwn}}^{2}(t) /(8 \pi)=\int \eta L\left(t^{\prime}\right) \mathrm{d} t^{\prime}$, and $R_{\mathrm{pwn}}$ is estimated by reproducing photon SED.

\subsection{Group 1: young PWNe}

There are six young PWNe in this group, N 158A, G21.5-0.9, Crab nebula, Kes 75, G310.6-1.6, and 3C 58, whose ages range from $700 \mathrm{yr}$ to $2400 \mathrm{yr}$. The parameters of pulsar and ejecta, electron injections, and soft photon fields for these young PWNe are listed in Table 1, and the details for each PWN is given in Appendix B. Using the parameters in Table 1, the SEDs of these young PWNe are calculated. The comparisons of the calculated and observed SEDs are shown in Fig. 1 and derived parameters are listed in Table 1. The multiband SEDs of these young PWNe have been studied (e.g., Tanaka \& Takahara 2011; Torres et al. 2014; Martin et al. 2014). These PWNe are in the SNR's free 
Table 2. Parameters for evolved PWNe.

\begin{tabular}{|c|c|c|c|c|c|c|}
\hline PWN & HESS J1813-178 & G54.1+0.3 & $\mathrm{G} 292.0+1.8$ & G0.9+0.1 & MSH 15-52 & N 157B \\
\hline \multicolumn{7}{|c|}{ Pulsar and ejecta parameters } \\
\hline$P(\mathrm{~ms})$ & 44.7 & 136.85 & 135.31 & 52.2 & 150 & 16.12 \\
\hline$\dot{P}\left(\mathrm{~s} \mathrm{~s}^{-1}\right)$ & $1.26 \times 10^{-13}$ & $7.51 \times 10^{-13}$ & $7.47 \times 10^{-13}$ & $1.56 \times 10^{-13}$ & $1.50 \times 10^{-12}$ & $5.13 \times 10^{-14}$ \\
\hline$n$ & 3.0 & 3.0 & 3.0 & 3.0 & 1.4 & 3.0 \\
\hline$\tau_{\mathrm{c}}(\mathrm{yr})$ & 5624 & 2889 & 2872 & 5305 & 1585 & 4982 \\
\hline$L(t)\left(\mathrm{erg} \mathrm{s}^{-1}\right)$ & $5.56 \times 10^{37}$ & $1.16 \times 10^{37}$ & $1.19 \times 10^{37}$ & $4.32 \times 10^{37}$ & $1.75 \times 10^{37}$ & $4.83 \times 10^{38}$ \\
\hline$T_{\text {age }}(\mathrm{yr})$ & 2500 & 2600 & 2700 & 3000 & 4000 & 4600 \\
\hline$L_{0}\left(\mathrm{erg} \mathrm{s}^{-1}\right)$ & $1.80 \times 10^{38}$ & $1.15 \times 10^{39}$ & $3.32 \times 10^{39}$ & $2.29 \times 10^{38}$ & $1.18 \times 10^{39}$ & $8.21 \times 10^{40}$ \\
\hline$\tau_{0}(\mathrm{yr})$ & 3124 & 289 & 172 & 2305 & 3927 & 382 \\
\hline$M_{\mathrm{ej}}\left(M_{\odot}\right)$ & 8.0 & 20.0 & 18.0 & 14.0 & 6.0 & 20.0 \\
\hline$E_{\mathrm{SN}}\left(10^{51} \mathrm{erg}\right)$ & 1.0 & 1.0 & 1.0 & 1.0 & 1.0 & 1.0 \\
\hline$n_{\mathrm{H}}\left(\mathrm{cm}^{3}\right)$ & 1.0 & 1.0 & 0.5 & 0.01 & 0.01 & 0.03 \\
\hline$d(\mathrm{kpc})$ & 4.7 & 7.0 & 6.0 & 13.3 & 4.4 & 53.7 \\
\hline \multicolumn{7}{|c|}{ Soft photon parameters } \\
\hline$T_{\text {FIR }}(\mathrm{K})$ & 40.0 & 25.0 & 25 & 30.0 & 30.0 & 50.0 \\
\hline$U_{\mathrm{FIR}}\left(\mathrm{eV} \mathrm{cm}^{-3}\right)$ & 0.1 & 0.8 & 1.0 & 3.8 & 1.2 & 5.0 \\
\hline$T_{\mathrm{NIR}}(\mathrm{K})$ & 4000.0 & 3000.0 & 2800 & 3000.0 & 3000.0 & 5000.0 \\
\hline$U_{\mathrm{NIR}}\left(\mathrm{eV} \mathrm{cm}^{-3}\right)$ & 0.5 & 1.1 & 0.7 & 25.0 & 2.2 & 4.0 \\
\hline \multicolumn{7}{|c|}{ Injection parameters } \\
\hline$E_{\mathrm{b}}\left(10^{5} \mathrm{MeV}\right)$ & 5.92 & 5.45 & 1.60 & 0.45 & 6.65 & 1.62 \\
\hline$\alpha_{1}$ & 1.09 & 1.22 & 1.56 & 1.10 & 1.17 & 1.15 \\
\hline$\alpha_{2}$ & $2.76 \pm 0.02$ & $2.67 \pm 0.03$ & $2.62 \pm 0.18$ & $2.52 \pm 0.02$ & $2.28 \pm 0.02$ & $2.58 \pm 0.01$ \\
\hline$\eta_{\mathrm{B}}$ & $0.0057 \pm 0.0008$ & $0.0045 \pm 0.0009$ & $0.046 \pm 0.007$ & $0.029 \pm 0.004$ & $0.45 \pm 0.03$ & $0.03 \pm 0.002$ \\
\hline$\epsilon$ & $0.26 \pm 0.05$ & $0.26 \pm 0.09$ & 0.42 & $0.25 \pm 0.08$ & $0.56 \pm 0.39$ & $0.33 \pm 0.05$ \\
\hline Chi-squared $\chi^{2}$ & 1.58 & 0.65 & 14.98 & 0.30 & 0.60 & 2.34 \\
\hline \multicolumn{7}{|c|}{ Derived parameters } \\
\hline$E_{\max }\left(10^{8} \mathrm{MeV}\right)$ & $7.59_{-1.91}^{+2.08}$ & $3.07_{-1.27}^{+1.46}$ & $16.13_{-1.28}^{+1.19}$ & $14.52_{-5.35}^{+6.00}$ & $81.42_{-57.52}^{-61.23}$ & $65.18_{-11.75}^{+12.35}$ \\
\hline$B_{\text {pwn }}(\mu \mathrm{G})$ & $8.11_{-0.75}^{+0.72}$ & $9.48_{-1.26}^{+1.21}$ & $23.98_{-2.23}^{+2.35}$ & $20.29_{-1.93}^{+1.86}$ & $18.72_{-0.37}^{+0.35}$ & $26.47_{-0.99}^{+0.94}$ \\
\hline$R_{\mathrm{pwn}}(\mathrm{pc})$ & 3.67 & 2.41 & 3.12 & 3.51 & 11.21 & 10.02 \\
\hline$R_{\mathrm{rs}}(\mathrm{pc})$ & 4.97 & 5.00 & 5.73 & 10.27 & 14.67 & 11.17 \\
\hline$R_{\mathrm{snr}}(\mathrm{pc})$ & 6.97 & 6.16 & 6.97 & 12.23 & 17.88 & 13.29 \\
\hline$R_{\mathrm{ts}}(\mathrm{pc})$ & 0.28 & 0.11 & 0.13 & 0.22 & 0.53 & 0.72 \\
\hline
\end{tabular}

expansion phases and the dynamical evolution in the free expansion phase used here is similar that used in Torres et al. (2014).

The fluxes, $F_{\mathrm{X}} \equiv F_{1-10 \mathrm{keV}}$ and $F_{\gamma} \equiv F_{1-10 \mathrm{TeV}}$, in X-ray (1-10 keV) and $\gamma$-ray $(1-10 \mathrm{TeV})$ bands for each PWN are calculated.

At an age of $760 \mathrm{yr} N 158 \mathrm{~A}$ was not detected in $\mathrm{GeV}$ and $\mathrm{TeV}$ $\gamma$-ray bands, so the sensitivity curves of the Cherenkov Telescope Array (CTA) is used as an upper limit to restrict soft photon parameters (see Table 1). Our results are shown in Table 1 and Fig. 1 , and give that $R_{\mathrm{pwn}}=0.68 \mathrm{pc}$ and $B_{\mathrm{pwn}}=45.22 \mu \mathrm{G}$, which is roughly consistent with the values given in Martin et al. (2014, $R_{\text {pwn }}=0.7 \mathrm{pc}$ and $\left.B_{\text {pwn }}=32 \mu \mathrm{G}\right)$. The observed SED can be reproduced and predicted as $F_{\mathrm{X}} / F_{\gamma} \sim 118$.

Our results for G21.5-0.9 are shown in Table 1 and Fig. 1. Here the age is assumed to be $T_{\text {age }}=900 \mathrm{yr}$, which is close to $T_{\text {age }}=870 \mathrm{yr}$ in Torres et al. (2014). Our results give $R_{\text {pwn }}=$ $0.86 \mathrm{pc}$ and $B_{\mathrm{pwn}} \approx 84 \mu \mathrm{G}$, which are consistent with those in Torres et al. $\left(2014 ; R_{\mathrm{pwn}}=0.9 \mathrm{pc}\right.$ and $\left.B_{\mathrm{pwn}}=71 \mu \mathrm{G}\right)$ and those in Tanaka \& Takahara $\left(2011 ; R_{\mathrm{pwn}}=1.0 \mathrm{pc}\right.$ and $B_{\mathrm{pwn}}=64$, and $47 \mu \mathrm{G})$. We note that $R_{\mathrm{pwn}}$ is less than that given in Abdalla et al. $\left(2017 ;<4\right.$ pc). The predicted $F_{\mathrm{X}} / F_{\gamma} \sim 130$.

For Crab nebulae, the results are the same as those given in Zhu et al. (2015). As mentioned above, the main differences between our results and those in Tanaka \& Takahara (2011) and Torres et al. (2014) come from the use of different synchrotron emissivity. Our results give $R_{\mathrm{pwn}} \approx 1.95 \mathrm{pc}(<3 \mathrm{pc}$ in Abdalla et al. 2017) and $B_{\text {pwn }} \approx 116 \mu \mathrm{G}$. The predicted $F_{\mathrm{X}} / F_{\gamma} \sim 460$.

For Kes 75, its age is assumed to be $1000 \mathrm{yr}$, which is slightly older than the ages given in Tanaka \& Takahara (2011) and Torres et al. (2014), and its SED is calculated with the same values of pulsar and ejecta parameters and soft photon parameters as those in Torres et al. (2014) and can reproduce the observed SED well with $F_{\mathrm{X}} / F_{\gamma} \sim 13$ (see Fig. 1). However, our injection parameters are different from those given in Torres et al. (2014) because the formula of synchrotron emissivity used here (see Eq. (12) of Zhang et al. 2008) is different from that used in Torres et al. (2014, see Eqs. (27) and (28) of Martin et al. 2012), resulting in different values of $R_{\mathrm{pwn}}=0.99 \mathrm{pc}$ and $B_{\mathrm{pwn}} \approx 14 \mu \mathrm{G}$ of Table 1 in comparison with those $\left(R_{\mathrm{pwn}}=0.9 \mathrm{pc}\right.$ and $B_{\mathrm{pwn}}=$ $19 \mu \mathrm{G})$ in Torres et al. (2014) and those $\left(R_{\mathrm{pwn}}=0.29 \mathrm{pc}\right.$ and $\left.B_{\text {pwn }}=20 \mu \mathrm{G}\right)$ in Tanaka \& Takahara (2011).

The HESS observation gives the upper limit of the flux at $\mathrm{TeV}$ band for G310.6-1.6, which can roughly restrict model parameters. With $T_{\text {age }}=1500 \mathrm{yr}$, which is less than the age of $<1900 \mathrm{yr}$ found in Renaud et al. (2010), $R_{\mathrm{pwn}}=1.32 \mathrm{pc}$ 

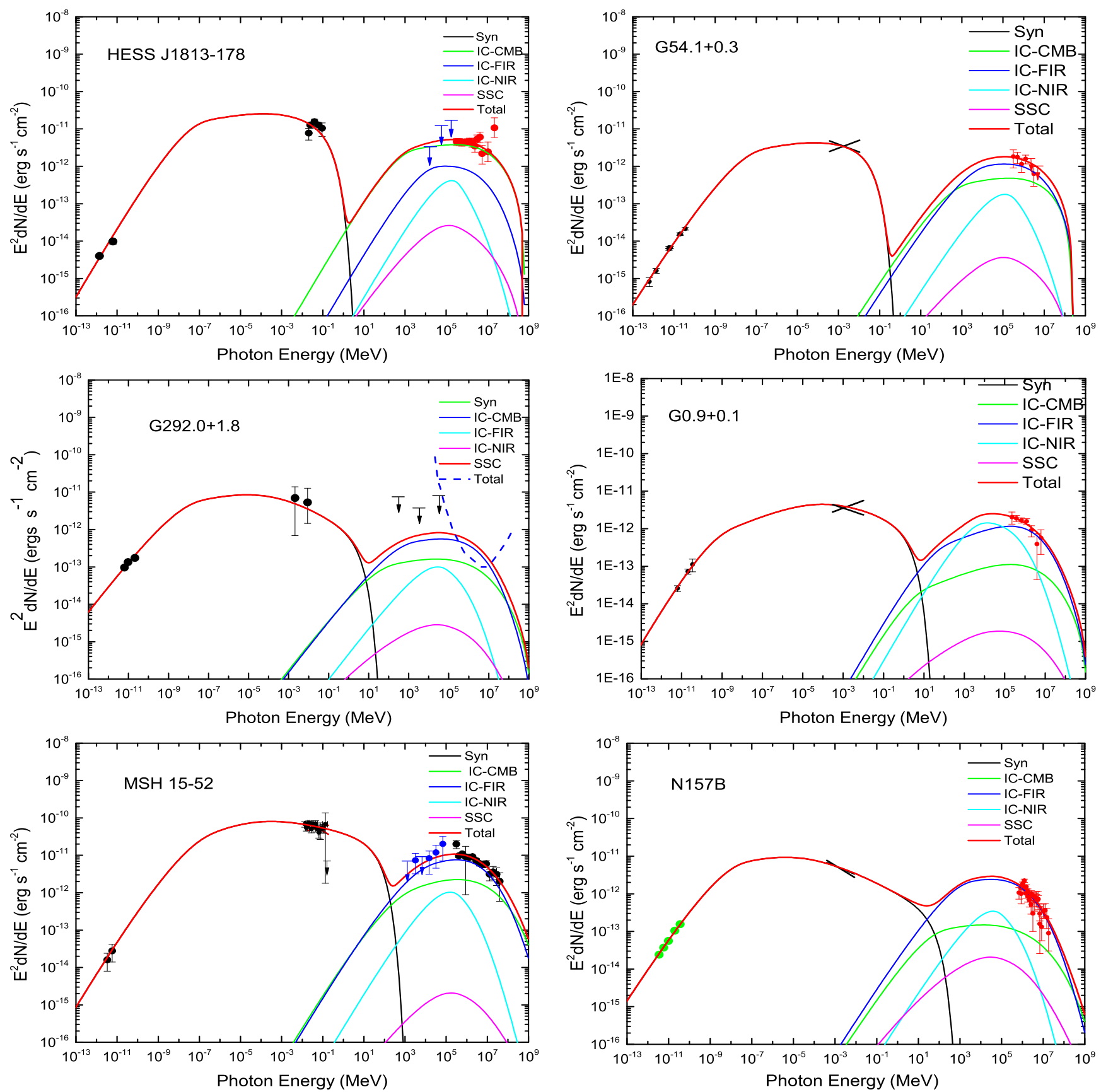

Fig. 2. Comparisons of predicted SEDs and observed data for evolved PWNe (from upper left panel for HEES J1813-178 to bottom right panel for N 157B). In each panel, the black line represents the synchrotron SED; magenta, blue, green, and cyan lines represent the SEDs of inverse Compton scatterings with the synchrotron photons, IR, CMB, and starlight, respectively; and the total SED is shown by the red line. See text for the descriptions of the observed data; the relevant parameters are listed in Table 2.

and $B_{\text {pwn }} \approx 13 \mu \mathrm{G}$ are obtained here. We note that Tanaka \& Takahara (2013) gave $R_{\text {pwn }}=1.3 \mathrm{pc}$ and $B_{\text {pwn }}=$ $17 \mu \mathrm{G}$ with $T_{\text {age }}=600 \mathrm{yr}$, and Martin et al. (2014) obtained $R_{\text {pwn }}=1.3 \mathrm{pc}$ and $B_{\text {pwn }}=8.2 \mu \mathrm{G}$ with $T_{\text {age }}=1100 \mathrm{yr}$. The predicted $F_{\mathrm{X}} / F_{\gamma} \sim 11$.

3C 58 has an age of $\sim 2400$ yr (Chevalier 2005; Tanaka \& Takahara 2013; Torres et al. 2013). With $T_{\text {age }}=$ $2400 \mathrm{yr}, R_{\mathrm{pwn}}=3.05 \mathrm{pc}$ and $B_{\mathrm{pwn}} \approx 24 \mu \mathrm{G}$ are obtained, which can compare with the values in Tanaka \& Takahara (2013, $R_{\mathrm{pwn}}=2.0 \mathrm{pc}$ and $\left.B_{\mathrm{pwn}}=17 \mu \mathrm{G}\right)$ and those in Torres et al. $\left(2013, R_{\mathrm{pwn}}=3.7 \mathrm{pc}\right.$ and $\left.B_{\mathrm{pwn}}=35 \mu \mathrm{G}\right)$. We note that the distance of the PWN is assumed to be $2 \mathrm{kpc}$ here, which is the same as that in Tanaka \& Takahara (2013), but is different from that in Torres et al. $(2013 ; 3.2 \mathrm{kpc})$. The predicted $F_{\mathrm{X}} / F_{\gamma} \sim 20$.

\subsection{Group 2: evolved PWNe}

The group of evolved PWNe consists of HESS J1813-178, G54.1+0.3, G292.0+1.8, G0.9+0.1, MSH 15-52, and N 157B whose ages range from $2500 \mathrm{yr}$ to $4600 \mathrm{yr}$. Since the SN explosion energy $E_{\mathrm{sn}}$ are unknown for these PWNe, here we assume $E_{\mathrm{sn}}=1.0 \times 10^{51} \mathrm{erg}$. The related parameters for the evolved PWNe are listed in Table 2 and the comparisons of the modeled and observed SEDs are shown in Fig. 2. 
Table 3. Parameters for mature/old PWNe.

\begin{tabular}{|c|c|c|c|c|c|c|}
\hline PWN & HESS J1356-645 & CTA 1 & HESS J1418-609 & HESS J1420-607 & HESS J1119-614 & HESS J1303-631 \\
\hline \multicolumn{7}{|l|}{ Pulsar and ejecta } \\
\hline$P(\mathrm{~ms})$ & 166 & 316.0 & 110.6 & 68.0 & 408.0 & 184 \\
\hline$\dot{P}\left(\mathrm{~s} \mathrm{~s}^{-1}\right)$ & $3.6 \times 10^{-13}$ & $3.614 \times 10^{-13}$ & $1.69 \times 10^{-13}$ & $8.3 \times 10^{-14}$ & $4.0 \times 10^{-12}$ & $2.65 \times 10^{-13}$ \\
\hline$n$ & 3.0 & 3.0 & 3.0 & 3.0 & 1.2 & 2.5 \\
\hline$\tau_{\mathrm{c}}(\mathrm{yr})$ & 7310 & 13863 & 10376 & 12990 & 1617 & 11008 \\
\hline$L(t)\left(\mathrm{erg} \mathrm{s}^{-1}\right)$ & $3.10 \times 10^{36}$ & $4.52 \times 10^{35}$ & $4.93 \times 10^{36}$ & $1.04 \times 10^{37}$ & $2.32 \times 10^{36}$ & $1.68 \times 10^{36}$ \\
\hline$T_{\text {age }}(\mathrm{yr})$ & 6500 & 7500 & 8000 & 8500 & 9000 & 13000 \\
\hline$L_{0}\left(\mathrm{erg} \mathrm{s}^{-1}\right)$ & $2.52 \times 10^{38}$ & $2.14 \times 10^{36}$ & $9.39 \times 10^{37}$ & $8.70 \times 10^{37}$ & $1.78 \times 10^{40}$ & $2.64 \times 10^{38}$ \\
\hline$\tau_{0}(\mathrm{yr})$ & 810 & 6363 & 2376 & 4490 & 7170 & 1678 \\
\hline$M_{\mathrm{ej}}\left(M_{\odot}\right)$ & 7.0 & 8.0 & 10.0 & 15.0 & 35.0 & 9.0 \\
\hline$E_{\mathrm{SN}}\left(10^{51} \mathrm{erg}\right)$ & 1.0 & 1.0 & 1.0 & 1.0 & 1.0 & 1.0 \\
\hline$n_{\mathrm{H}}\left(\mathrm{cm}^{3}\right)$ & 0.05 & 0.1 & 0.2 & 0.5 & 0.06 & 0.02 \\
\hline$d(\mathrm{kpc})$ & 2.5 & 1.4 & 5.0 & 5.6 & 8.4 & 6.6 \\
\hline \multicolumn{7}{|c|}{ Soft photon parameters } \\
\hline$T_{\text {FIR }}(\mathrm{K})$ & 25.0 & 25.0 & 40.0 & 40.0 & 25.0 & 40.0 \\
\hline$U_{\mathrm{FIR}}\left(\mathrm{eV} \mathrm{cm}^{-3}\right)$ & 0.4 & 0.3 & 0.4 & 0.3 & 0.3 & 6.0 \\
\hline$T_{\mathrm{NIR}}(\mathrm{K})$ & 5000.0 & 3000.0 & 4000.0 & 4000.0 & 3300.0 & 4000.0 \\
\hline$U_{\text {NIR }}\left(\mathrm{eV} \mathrm{cm}^{-3}\right)$ & 0.5 & 0.6 & 1.0 & 0.3 & 0.7 & 2.0 \\
\hline \multicolumn{7}{|c|}{ Injection parameters } \\
\hline$E_{\mathrm{b}}\left(10^{5} \mathrm{MeV}\right)$ & 5.02 & 2.00 & 6.00 & 7.00 & 7.70 & 36.00 \\
\hline$\alpha_{1}$ & 1.05 & 1.20 & 1.15 & 1.35 & 1.50 & 1.50 \\
\hline$\alpha_{2}$ & $2.68 \pm 0.02$ & $2.11 \pm 0.11$ & $2.52 \pm 0.08$ & $2.73 \pm 0.06$ & $3.08 \pm 0.09$ & $2.19 \pm 0.02$ \\
\hline$\eta_{\mathrm{B}}$ & $0.11 \pm 0.03$ & $0.49 \pm 0.02$ & $0.058 \pm 0.015$ & $0.011 \pm 0.002$ & $0.0010 \pm 0.0001$ & 0.07 \\
\hline$\epsilon$ & $0.17 \pm 0.08$ & $0.20 \pm 0.12$ & $0.38 \pm 0.35$ & $0.34 \pm 0.16$ & $0.69 \pm 0.01$ & 0.12 \\
\hline Chi-squared $\chi^{2}$ & 1.39 & 4.25 & 0.92 & 0.40 & 2.48 & 3.94 \\
\hline \multicolumn{7}{|c|}{ Derived parameters } \\
\hline$E_{\max }\left(10^{8} \mathrm{MeV}\right)$ & $5.15_{-2.83}^{+3.39}$ & $4.88_{-2.97}^{+3.08}$ & $10.54_{-9.82}^{+12.17}$ & $5.97_{-3.11}^{+3.57}$ & $1.72_{-0.11}^{+0.12}$ & 2.13 \\
\hline$B_{\mathrm{pwn}}(\mu \mathrm{G})$ & $4.31_{-0.59}^{+0.48}$ & $5.66_{-0.23}^{+0.27}$ & $4.38_{-0.63}^{+0.60}$ & $3.73_{-0.40}^{+0.38}$ & $1.34_{-0.07}^{+0.07}$ & 1.63 \\
\hline$R_{\mathrm{pwn}}(\mathrm{pc})$ & 10.34 & 6.24 & 9.43 & 7.73 & 14.67 & 20.44 \\
\hline$R_{\mathrm{rs}}(\mathrm{pc})$ & 13.06 & 10.84 & 0 & 0 & 0 & 0 \\
\hline$R_{\mathrm{snr}}(\mathrm{pc})$ & 18.72 & 17.67 & 15.90 & 13.59 & 17.00 & 30.17 \\
\hline$R_{\mathrm{ts}}(\mathrm{pc})$ & 0.56 & 0.28 & 0.47 & 0.34 & 0.16 & 1.22 \\
\hline
\end{tabular}

HESS J1813-178 is observed to have a distance $d \approx 4.7 \mathrm{kpc}$ (Brogan et al. 2005; Halpern et al. 2012) and $R_{\text {pwn }}=4.0 \pm 0.3 \mathrm{pc}$ (Abdalla et al. 2017). With $T_{\text {age }}=2500 \mathrm{yr}, R_{\mathrm{pwn}} \approx 3.7 \mathrm{pc}$ and $B_{\text {pwn }}=8.11 \mu \mathrm{G}$ are obtained here. The predicted $F_{\mathrm{X}} / F_{\gamma} \sim 6$. We note that Fang \& Zhang (2010b) investigated the PWN in detail, and obtained $R_{\mathrm{pwn}}=1.7 \mathrm{pc}$ and $B_{\mathrm{pwn}}=5 \mu \mathrm{G}$ with $T_{\mathrm{age}}=$ $1200 \mathrm{yr}$.

The age of G54.1+0.3 is uncertain, which ranges from 1500 to $6000 \mathrm{yr}$ (Camilo et al. 2002a), or from 2100 to $3600 \mathrm{yr}$ (Gelfand et al. 2015). Because the characteristic age is $\sim 2872 \mathrm{yr}$, $T_{\text {age }}=2600 \mathrm{yr}$ is assumed, and our results give $R_{\text {pwn }} \sim$ $2.4 \mathrm{pc}, B_{\mathrm{pwn}} \approx 9.5 \mu \mathrm{G}$, and $F_{\mathrm{X}} / F_{\gamma} \sim 3$. With different ages, Tanaka \& Takahara (2011) gave $R_{\mathrm{pwn}}=1.8 \mathrm{pc}$ and $B_{\mathrm{pwn}}=$ $10 \mu \mathrm{G}(6.7 \mu \mathrm{G})$ for $T_{\text {age }}=1700 \mathrm{yr}(2300 \mathrm{yr})$ and Torres et al. (2014) obtained $R_{\text {pwn }}=1.4 \mathrm{pc}$ and $B_{\mathrm{pwn}}=14 \mu \mathrm{G}$ for $T_{\mathrm{age}}=$ 1700 yr.

Although the age of $\mathrm{G} 292.0+1.8$ is uncertain, $R_{\mathrm{pwn}} \approx$ $3.0 \mathrm{pc}$ is given in Bhalerao et al. (2015). With $T_{\text {age }}=2700 \mathrm{yr}$ (Tanaka \& Takahara 2013), which is larger than $1600 \mathrm{yr}$ given in Murdin \& Clark (1979) and $2500 \mathrm{yr}$ in Martin et al. (2014), our results show that $R_{\mathrm{pwn}}=3.12 \mathrm{pc}$ and $B_{\mathrm{pwn}} \approx 24 \mu \mathrm{G}$, which are consistent with $R_{\mathrm{pwn}}=3.5 \mathrm{pc}$ and $B_{\mathrm{pwn}}=16 \mu \mathrm{G}$ obtained by Tanaka \& Takahara (2013) and $R_{\mathrm{pwn}}=3.5 \mathrm{pc}$ and $B_{\mathrm{pwn}}=21 \mu \mathrm{G}$ by Martin et al. (2014). The predicted $F_{\mathrm{X}} / F_{\gamma} \sim 14$.

The age (from $2000 \mathrm{yr}$ to $3000 \mathrm{yr}$, see Camilo et al. 2009) and distance (from $8.0 \mathrm{kpc}$ to $16 \mathrm{kpc}$, see Dubner et al. 2008) of G0.9+0.1 are both uncertain. Here $T_{\text {age }}=3000 \mathrm{yr}$ and $d=13.3 \mathrm{kpc}$ are assumed; the results show that $R_{\mathrm{pwn}}=3.51 \mathrm{pc}$, $B_{\text {pwn }} \approx 20 \mu \mathrm{G}$, and $F_{\mathrm{X}} / F_{\gamma} \sim 4$. Tanaka \& Takahara (2011) obtained $R_{\mathrm{pwn}}=2.3 \mathrm{pc}(3.8 \mathrm{pc})$ and $B_{\mathrm{pwn}}=15 \mu \mathrm{G}(12 \mu \mathrm{G})$ for $d=8.0 \mathrm{kpc}(13 \mathrm{kpc})$ and $T_{\mathrm{age}}=2000 \mathrm{yr}(4500 \mathrm{yr})$. Torres et al. (2014) obtained $R_{\text {pwn }}=2.5 \mathrm{pc}(3.8 \mathrm{pc})$ and $B_{\mathrm{pwn}}=14 \mu \mathrm{G}$ $(15 \mu \mathrm{G})$ for $d=8.5 \mathrm{kpc}(13 \mathrm{kpc})$ and $T_{\mathrm{age}}=2000 \mathrm{yr}(3000 \mathrm{yr})$.

For MSH 15-52, its radius is $R_{\text {pwn }}=11.1 \pm 2.0 \mathrm{pc}$ (Abdalla et al. 2017). Our results give $R_{\mathrm{pwn}}=11.21 \mathrm{pc}, B_{\mathrm{pwn}} \approx$ $19 \mu \mathrm{G}$, and $F_{\mathrm{X}} / F_{\gamma} \sim 10$ with $T_{\text {age }}=4000 \mathrm{yr}$. We note that younger age in previous studies is used; for example, $T_{\text {age }} \sim$ $1600 \mathrm{yr}, R_{\mathrm{pwn}}=3.0 \mathrm{pc}$, and $B_{\mathrm{pwn}}=21 \mu \mathrm{G}$ were obtained by Torres et al. (2014), and $R_{\mathrm{pwn}}=3.6 \mathrm{pc}$ and $B_{\mathrm{pwn}}=19.0 \mu \mathrm{G}$ by Fang \& Zhang (2010a).

$\mathrm{N} 157 \mathrm{~B}$ has an estimated distance of $48 \mathrm{kpc}$ (Macri et al. 2006), and its radius is about $10.6 \mathrm{pc}$ (Lazendic et al. 2000), or 3.5 pc (Chen et al. 2006), or $<94$ pc (Abdalla et al. 2017). Here, $T_{\text {age }}=4600 \mathrm{yr}$ is used, and the results show that $R_{\mathrm{pwn}}=$ $10.02 \mathrm{pc}, B_{\mathrm{pwn}} \approx 26 \mu \mathrm{G}$, and $F_{\mathrm{X}} / F_{\gamma} \sim 5$. With $T_{\mathrm{age}}=4600 \mathrm{yr}$, Martin et al. (2014) obtained $R_{\text {pwn }}=10.6$ pc and $B_{\text {pwn }}=13 \mu \mathrm{G}$.

\subsection{Group 3: mature/old PWNe}

The third group of mature/old PWNe consists of HESS J1356645, CTA 1, HESS J1418-609, HESS J1420-607, HESS J1119614, and HESS J1303-631 whose ages range from $6500 \mathrm{yr}$ to 

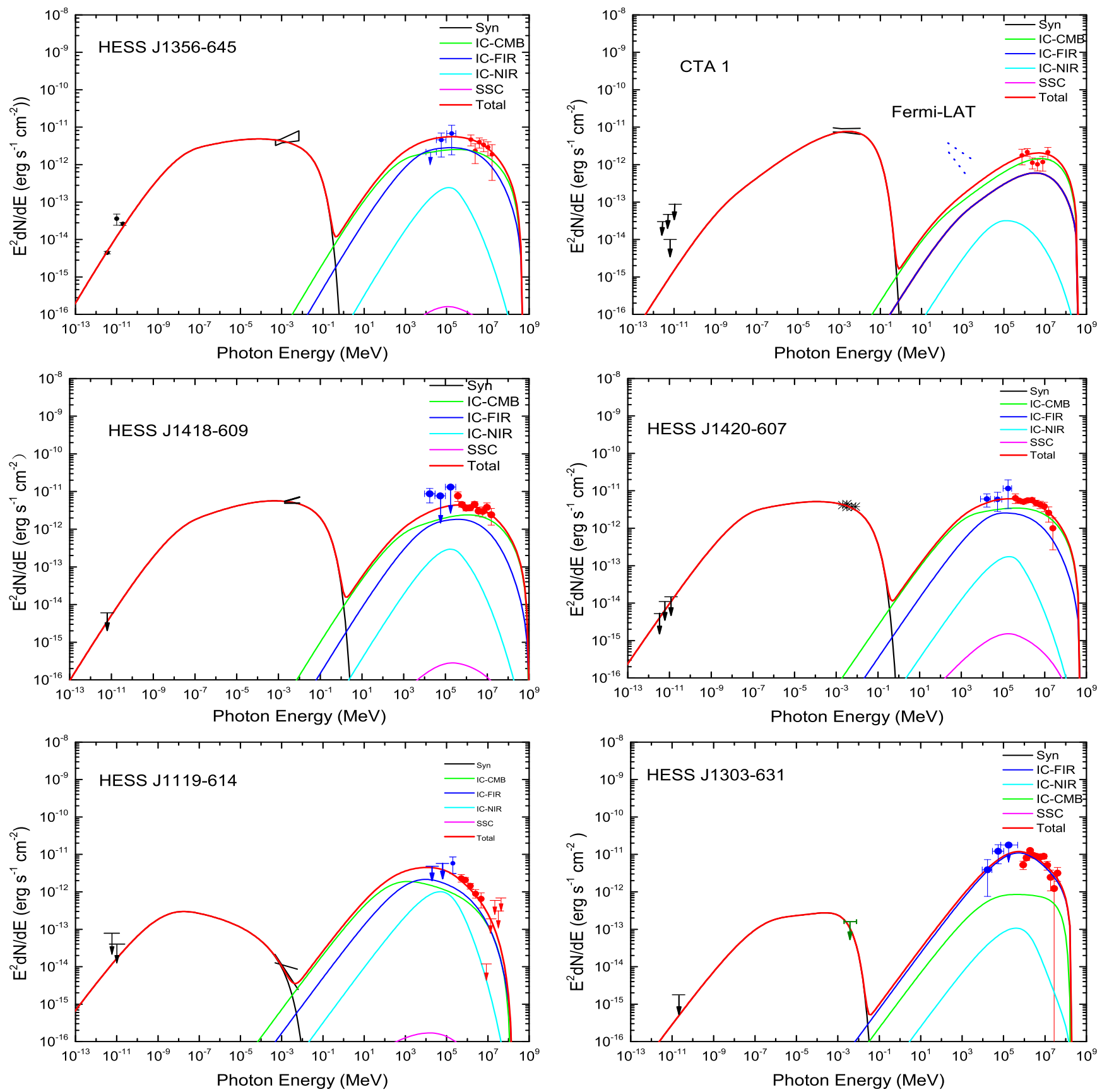

Fig. 3. Comparisons of predicted SEDs and observed data for mature/old PWNe (from upper left panel for HEES J1356-645 to bottom right panel for HESS J1303-631). In each panel, the black line represents the synchrotron SED; magenta, blue, green, and cyan lines represent the SEDs of inverse Compton scatterings with the synchrotron photons, IR, CMB, and starlight, respectively; and the total SED is shown by the red line. See text for the descriptions of the observed data; the relevant parameters are listed in Table 3.

13000 yr. The related parameters for these mature/old PWNe are listed in Table 3 and the comparisons of modeled and observed SEDs are shown in Fig. 3.

HESS J1356-645 is a TeV PWN with a distance of $2.5 \mathrm{kpc}$ (Cordes \& Lazio 2002; Chang et al. 2012) and a radius of $R_{\mathrm{pwn}}=$ $10.1 \pm 0.9 \mathrm{pc}$ (Abdalla et al. 2017). With $T_{\text {age }}=6500 \mathrm{yr}$, the values of $R_{\mathrm{pwn}}=10.34 \mathrm{pc}, B_{\mathrm{pwn}} \approx 4 \mu \mathrm{G}$, and $F_{\mathrm{X}} / F_{\gamma} \sim 0.9$ are obtained. With $T_{\text {age }}=6000(8000) \mathrm{yr}$, Torres et al. (2014) obtained $R_{\text {pwn }}=9.5(9.9)$ pc and $B_{\text {pwn }} \approx 3.1(3.5) \mu \mathrm{G}$.

CTA 1 has a radius of $R_{\mathrm{pwn}}=6.6 \pm 0.5 \mathrm{pc}$ (Abdalla et al. 2017). With $T_{\mathrm{age}}=7500 \mathrm{yr}$, the values of $R_{\mathrm{pwn}}=6.24 \mathrm{pc}$, $B_{\text {pwn }} \approx 5.66 \mu \mathrm{G}$, and $F_{\mathrm{X}} / F_{\gamma} \sim 4$ are obtained here. There have been several studies of CTA 1 (e.g., Zhang et al. 2009;
Torres et al. 2014; Martin et al. 2016). Torres et al. (2014) found $R_{\text {pwn }}=8 \mathrm{pc}$ and $B_{\text {pwn }} \approx 4.1 \mu \mathrm{G}$ with $T_{\text {age }}=7500 \mathrm{yr}$. Martin et al. (2016) studied both radiative and dynamical properties of CTA 1; they obtained that $T_{\text {age }}=9200(11400) \mathrm{yr}$, $R_{\text {pwn }}=6.7(6.7) \mathrm{pc}$, and $B_{\text {pwn }} \approx 4.3(1.8) \mu \mathrm{G}$ in the free expansion (compression) phase of CTA 1.

For HESS J1418-609, its radius is $R_{\mathrm{pwn}}=9.4 \pm 0.9 \mathrm{pc}$ (Abdalla et al. 2017). In the current paper $R_{\mathrm{pwn}}=9.4 \mathrm{pc}, B_{\mathrm{pwn}} \approx$ $4.4 \mu \mathrm{G}$, and $F_{\mathrm{X}} / F_{\gamma} \sim 1$ are obtained with $T_{\text {age }}=8000 \mathrm{yr}$.

The radius of HESS $\mathrm{J} 1420-607$ is $R_{\mathrm{pwn}}=7.9 \pm 0.6 \mathrm{pc}$ (Abdalla et al. 2017). Here the values of $R_{\mathrm{pwn}}=7.73 \mathrm{pc}, B_{\mathrm{pwn}} \approx$ $3.7 \mu \mathrm{G}$, and $F_{\mathrm{X}} / F_{\gamma} \sim 0.8$ are obtained with $T_{\text {age }}=8500 \mathrm{yr}$. 
B.-T. Zhu et al.: Multiband nonthermal radiative properties of pulsar wind nebulae

Table 4. Pair multiplicities and wind Lorentz factors in the sample.

\begin{tabular}{lccc}
\hline \hline PWN & $Q\left(\mathrm{~s}^{-1}\right)$ & $\kappa_{\text {multi }}$ & $\gamma_{\omega}$ \\
\hline Young PWNe & & & \\
\hline N158A & $4.48 \times 10^{41}$ & $1.02 \times 10^{8}$ & $4.01 \times 10^{2}$ \\
G21.5-0.9 & $6.76 \times 10^{38}$ & $3.21 \times 10^{5}$ & $5.83 \times 10^{4}$ \\
Crab nebula & $5.93 \times 10^{41}$ & $7.73 \times 10^{7}$ & $8.97 \times 10^{2}$ \\
Kes 75 & $4.02 \times 10^{39}$ & $3.87 \times 10^{6}$ & $2.49 \times 10^{3}$ \\
G310.6-1.6 & $4.42 \times 10^{38}$ & $1.71 \times 10^{5}$ & $1.40 \times 10^{5}$ \\
3C 58 & $1.88 \times 10^{39}$ & $1.00 \times 10^{6}$ & $1.64 \times 10^{4}$ \\
\hline Evolved PWNe & & & \\
\hline HESS J1813-178 & $6.82 \times 10^{38}$ & $2.52 \times 10^{5}$ & $9.91 \times 10^{4}$ \\
G54.1+0.3 & $4.34 \times 10^{38}$ & $3.52 \times 10^{5}$ & $3.25 \times 10^{4}$ \\
G292.0+1.8 & $2.42 \times 10^{40}$ & $1.93 \times 10^{7}$ & $6.33 \times 10^{3}$ \\
G0.9+0.1 & $4.13 \times 10^{39}$ & $1.73 \times 10^{6}$ & $5.73 \times 10^{2}$ \\
MSH 15-52 & $1.11 \times 10^{38}$ & $7.31 \times 10^{4}$ & $1.06 \times 10^{5}$ \\
N157B & $2.38 \times 10^{40}$ & $2.98 \times 10^{6}$ & $2.41 \times 10^{4}$ \\
\hline Mature/old PWNe & & & \\
\hline HESS J1356-645 & $2.75 \times 10^{37}$ & $4.30 \times 10^{4}$ & $1.23 \times 10^{6}$ \\
CTA 1 & $4.25 \times 10^{36}$ & $1.74 \times 10^{4}$ & $6.63 \times 10^{4}$ \\
HESS J1418-609 & $7.46 \times 10^{37}$ & $9.26 \times 10^{4}$ & $7.61 \times 10^{4}$ \\
HESS J1420-607 & $1.25 \times 10^{39}$ & $1.07 \times 10^{6}$ & $1.01 \times 10^{4}$ \\
HESS J1119-614 & $1.57 \times 10^{39}$ & $2.84 \times 10^{6}$ & $1.81 \times 10^{3}$ \\
HESS J1303-631 & $1.96 \times 10^{38}$ & $4.17 \times 10^{5}$ & $9.75 \times 10^{3}$ \\
\hline
\end{tabular}

HESS J1119-614 has a radius of $R_{\mathrm{pwn}}=14 \pm 2 \mathrm{pc}$ (Abdalla et al. 2017). The values reported here are $R_{\mathrm{pwn}}=$ $14.67 \mathrm{pc}, B_{\mathrm{pwn}} \approx 1.3 \mu \mathrm{G}$, and $F_{\mathrm{X}} / F_{\gamma} \sim 0.01$ with $T_{\text {age }}=9000 \mathrm{yr}$ here. Torres et al. (2014) gave $R_{\text {pwn }}=13 \mathrm{pc}$ and $B_{\text {pwn }} \approx 4 \mu \mathrm{G}$ with $T_{\text {age }}=4200 \mathrm{yr}$.

HESS J1303-631 has a radius of $R_{\mathrm{pwn}}=20.6 \pm 1.7 \mathrm{pc}$ (Abdalla et al. 2017). In the current paper $R_{\mathrm{pwn}}=20.44 \mathrm{pc}$, $B_{\text {pwn }} \approx 1.6 \mu \mathrm{G}$ and $F_{\mathrm{X}} / F_{\gamma} \sim 0.02$ are obtained with $T_{\text {age }}=$ $13000 \mathrm{yr}$.

\subsection{Estimate of pair multiplicity and wind Lorentz factor}

Pair multiplicity, $\kappa_{\text {multi }}$, and the Lorentz factor, $\gamma_{\mathrm{w}}$, are calculated for a PWN based on our model. The pair multiplicity can be expressed as

$\kappa_{\text {multi }}=\frac{Q}{\dot{N}}$,

where $Q$ is the injection rate by integrating Eq. (2),

$Q=\int_{E_{\min }}^{E_{\max }} Q(E, t) \mathrm{d} E$,

and $\dot{N}$ is the injection rate with electrodynamic minimum suggested by Goldreich \& Julian (1969), i.e., $\dot{N}=\left(\frac{c I \Omega \dot{\Omega}}{e^{2}}\right)^{1 / 2}$. The values are shown in Table 5 . The pair multiplicity ranges from $\sim 10^{5}$ to $\sim 10^{8}$. The Lorentz factor of the pulsar wind can be estimated by

$\langle E\rangle=\frac{\left(1-\eta_{\mathrm{B}}\right) L(t)}{\int Q(E, t) \mathrm{d} E}=\gamma_{w} m_{\mathrm{e}} c^{2}$,
Table 5. Parameters of the central pulsars in the sample.

\begin{tabular}{lccc}
\hline \hline PWN & \multicolumn{1}{c}{$B_{\mathrm{S}}$} & $\Phi$ & $B_{\mathrm{LC}}$ \\
\hline Young PWNe & & & \\
\hline N158A & $9.95 \times 10^{12}$ & $2.56 \times 10^{16}$ & $7.12 \times 10^{5}$ \\
G21.5-0.9 & $7.15 \times 10^{12}$ & $1.23 \times 10^{16}$ & $2.79 \times 10^{5}$ \\
Crab nebula & $7.61 \times 10^{12}$ & $4.47 \times 10^{16}$ & $1.88 \times 10^{6}$ \\
Kes 75 & $9.69 \times 10^{13}$ & $6.05 \times 10^{15}$ & $2.63 \times 10^{4}$ \\
G310.6-1.6 & $2.23 \times 10^{12}$ & $1.50 \times 10^{16}$ & $6.78 \times 10^{5}$ \\
3C 58 & $7.21 \times 10^{12}$ & $1.09 \times 10^{16}$ & $2.34 \times 10^{5}$ \\
\hline Evolved PWNe & & & \\
\hline HESS J1813-178 & $4.79 \times 10^{12}$ & $1.57 \times 10^{16}$ & $4.95 \times 10^{5}$ \\
G54.1+0.3 & $2.05 \times 10^{13}$ & $7.19 \times 10^{15}$ & $7.38 \times 10^{4}$ \\
G292.0+1.8 & $2.03 \times 10^{13}$ & $7.29 \times 10^{15}$ & $7.57 \times 10^{4}$ \\
G0.9+0.1 & $5.77 \times 10^{12}$ & $1.39 \times 10^{16}$ & $3.74 \times 10^{5}$ \\
MSH 15-52 & $3.03 \times 10^{13}$ & $8.85 \times 10^{15}$ & $8.29 \times 10^{4}$ \\
N157B & $1.84 \times 10^{12}$ & $4.64 \times 10^{16}$ & $4.05 \times 10^{6}$ \\
\hline Mature/old PWNe & & & \\
\hline HESS J1356-645 & $1.56 \times 10^{13}$ & $3.73 \times 10^{15}$ & $3.15 \times 10^{4}$ \\
CTA 1 & $2.16 \times 10^{13}$ & $1.42 \times 10^{15}$ & $6.32 \times 10^{3}$ \\
HESS J1418-609 & $8.75 \times 10^{12}$ & $4.69 \times 10^{15}$ & $5.96 \times 10^{4}$ \\
HESS J1420-607 & $4.81 \times 10^{12}$ & $6.82 \times 10^{15}$ & $1.41 \times 10^{5}$ \\
HESS J1119-614 & $8.17 \times 10^{13}$ & $3.22 \times 10^{15}$ & $1.11 \times 10^{4}$ \\
HESS J1303-631 & $1.41 \times 10^{13}$ & $2.74 \times 10^{15}$ & $2.09 \times 10^{4}$ \\
\hline & & &
\end{tabular}

where $\langle E\rangle$ is the average energy for the SED of each PWN. The values of $\gamma_{\mathrm{w}}$ are listed in Table 4. Clearly, $E_{\mathrm{b}}$ is larger than $\langle E\rangle$ by up to several orders of magnitude.

\subsection{Brief summary of calculated results}

Here, the main results in the above calculations are briefly summarized.

The observed SEDs of 18 PWNe can be reproduced well in the frame of the model. For the electron injection, five free parameters $\left(\alpha_{1}, \alpha_{2}, E_{\mathrm{b}}, \eta_{\mathrm{B}}, \epsilon\right)$ are used. Our results give $\alpha_{1} \approx 1.0$ $1.8, \alpha_{2} \approx 2.0-3.1, E_{\mathrm{b}} \approx 10^{4}-10^{7} \mathrm{MeV}, \eta_{\mathrm{B}} \approx 0.1 \%-11 \%$ for the PWNe except for MSH 15-52 $\left(\eta_{\mathrm{B}} \approx 45 \%\right)$ and CTA 1 $\left(\eta_{\mathrm{B}} \approx 49 \%\right.$ ), and $\epsilon \approx 0.1-0.70$. The results for spectral indices are consistent with previous studies (e.g., Bucciantini et al. 2011; Tanaka \& Takahara 2011; Torres et al. 2014). According to Sironi \& Spitkovsky (2011, 2012, 2013), these low-energy electrons are accelerated by relativistic magnetic reconnection in a PWN; its spectral slope in the range at 1.0-2.0. Therefore, the low-energy electron component possibly originates from relativistic magnetic reconnection. The high-energy electron component with spectral slope from 2.0-3.0 are consistent with that produced by the Fermi-type process (e.g., Achterberg et al. 2001); thus, high-energy electrons are from the Fermi-type process in the termination shock. The PWN magnetization is weak, which means that most PWNe are particle-dominated. This conclusion is consistent with result of Torres et al. (2014). The values of $\epsilon$ are in the range 0.1-0.45, except for HESS J1119-614, and of the break energy $E_{\mathrm{b}}$ are in the range $10^{4}-10^{7} \mathrm{MeV}$.

The fluxes, $F_{\mathrm{X}} \equiv F_{1-10 \mathrm{keV}}$ and $F_{\gamma} \equiv F_{1-10 \mathrm{TeV}}$, in X-ray (1$10 \mathrm{keV})$ and $\gamma$-ray $(1-10 \mathrm{TeV})$ bands for each PWN are obtained from our calculations. Our results show that $F_{\mathrm{X}} / F_{\gamma}>10$ for 
Table 6. Calculated luminosity at different wave bands and synchrotron cooling break energies in the sample.

\begin{tabular}{|c|c|c|c|c|c|c|}
\hline PWN & $\begin{array}{c}L_{\mathrm{R}} \\
(1.4 \mathrm{GHz}) \\
\mathrm{erg} / \mathrm{s}\end{array}$ & $\begin{array}{c}L_{\mathrm{X}} \\
(1-10 \mathrm{keV}) \\
\mathrm{erg} / \mathrm{s}\end{array}$ & $\begin{array}{c}L_{\gamma} \\
(1-10 \mathrm{TeV}) \\
\mathrm{erg} / \mathrm{s}\end{array}$ & $\mathrm{erg} / \mathrm{s}$ & $\begin{array}{c}E_{\mathrm{s}} \\
\mathrm{MeV}\end{array}$ & $\mathrm{erg} / \mathrm{s}$ \\
\hline \multicolumn{7}{|l|}{ Young PWNe } \\
\hline N158A & $3.32_{-1.40}^{+1.13} \times 10^{32}$ & $1.10_{-0.27}^{+0.27} \times 10^{37}$ & $9.28_{-4.64}^{+2.75} \times 10^{34}$ & $3.94_{-0.98}^{+0.05} \times 10^{37}$ & $5.68_{-3.25}^{+5.98} \times 10^{-5}$ & $2.35_{-0.95}^{+0.33} \times 10^{36}$ \\
\hline G21.5-0.9 & $2.38_{-0.44}^{+0.53} \times 10^{32}$ & $3.65_{-0.31}^{+0.30} \times 10^{35}$ & $2.81_{-0.39}^{+0.50} \times 10^{33}$ & $3.67_{-0.36}^{+0.35} \times 10^{36}$ & $6.24_{-1.35}^{+2.02} \times 10^{-6}$ & $2.79_{-0.29}^{+0.27} \times 10^{35}$ \\
\hline Crab nebula & $6.90_{-0.20}^{+0.20} \times 10^{33}$ & $1.81_{-0.06}^{+0.06} \times 10^{37}$ & $3.94_{-0.13}^{+0.13} \times 10^{34}$ & $1.34_{-0.03}^{+0.03} \times 10^{38}$ & $2.18_{-0.05}^{+0.06} \times 10^{-6}$ & $9.18_{-0.09}^{+0.02} \times 10^{36}$ \\
\hline Kes 75 & $2.06_{-0.96}^{+1.42} \times 10^{31}$ & $1.73_{-0.67}^{+0.37} \times 10^{35}$ & $1.37_{-0.51}^{+0.55} \times 10^{34}$ & $5.76_{-2.38}^{+1.97} \times 10^{35}$ & $1.06_{-0.50}^{+0.94} \times 10^{-3}$ & $5.72_{-1.75}^{+1.02} \times 10^{34}$ \\
\hline G310.6-1.6 & $1.45_{-0.16}^{+0.15} \times 10^{31}$ & $1.93_{-0.60}^{+0.80} \times 10^{35}$ & $1.89_{-0.34}^{+0.40} \times 10^{34}$ & $1.12_{-0.24}^{+0.29} \times 10^{36}$ & $5.92_{-1.06}^{+1.42} \times 10^{-4}$ & $9.06_{-2.59}^{+3.19} \times 10^{34}$ \\
\hline $3 C 58$ & $2.18_{-0.28}^{+0.31} \times 10^{32}$ & $5.16_{-1.55}^{+1.03} \times 10^{33}$ & $2.58_{-0.48}^{+0.56} \times 10^{32}$ & $1.85_{-0.28}^{+0.28} \times 10^{35}$ & $3.84_{-0.72}^{+1.03} \times 10^{-5}$ & $6.68_{-1.41}^{+1.56} \times 10^{33}$ \\
\hline \multicolumn{7}{|l|}{ Evolved PWNe } \\
\hline HESS & $3.43_{-0}^{+0}$ & $1.55_{-0.30}^{+0.31} \times 10^{35}$ & $2.78_{-0.34}^{+0.40} \times 10^{34}$ & $1.06_{-0.18}^{+0.19} \times 10^{36}$ & $9.10_{-2.06}^{+3.05} \times 10^{-4}$ & $6.28_{-1.24}^{+1.34} \times 10^{34}$ \\
\hline $\mathrm{G} 54.1+0.3$ & $3.39_{-0.84}^{+1.01} \times 10^{31}$ & $4.91_{-2.01}^{+1.91} \times 10^{34}$ & $1.79_{-0.32}^{+0.42} \times 10^{34}$ & $4.22_{-1.02}^{+1.12} \times 10^{35}$ & $5.26_{-1.59}^{+2.82} \times 10^{-4}$ & $2.30_{-0.76}^{+0.77} \times 10^{34}$ \\
\hline $\mathrm{G} 292.0+1.8$ & $3.92_{-0.70}^{+0.60} \times 10^{32}$ & $5.54_{-3.64}^{+9.73} \times 10^{34}$ & $4.02_{-2.25}^{+4.86} \times 10^{33}$ & $5.82_{-2.45}^{+4.98} \times 10^{35}$ & $3.01_{-0.74}^{+1.03} \times 10^{-5}$ & $3.54_{-1.90}^{+3.26} \times 10^{34}$ \\
\hline G0.9+0.1 & $5.60_{-1.13}^{+1.36} \times 10^{32}$ & $2.11_{-0.37}^{+0.39} \times 10^{35}$ & $5.47_{-0.94}^{+1.20} \times 10^{34}$ & $1.85_{-0.29}^{+0.32} \times 10^{36}$ & $4.03_{-0.93}^{+1.41} \times 10^{-5}$ & $9.31_{-1.55}^{+1.67} \times 10^{34}$ \\
\hline MSH 15-52 & $6.88_{-2.10}^{+3.01} \times 10^{31}$ & $4.89_{-0.57}^{+0.57} \times 10^{35}$ & $4.88_{-0.66}^{+0.72} \times 10^{34}$ & $3.14_{-0.54}^{+0.49} \times 10^{36}$ & $2.89_{-0.16}^{+0.18} \times 10^{-5}$ & $1.69_{-0.18}^{+0.19} \times 10^{35}$ \\
\hline N157B & $1.33_{-0.10}^{+0.11} \times 10^{34}$ & $4.31_{-0.30}^{+0.31} \times 10^{36}$ & $8.64_{-0.65}^{+0.70} \times 10^{35}$ & $5.52_{-0.32}^{+0.32} \times 10^{37}$ & $7.72_{-0.77}^{+0.92} \times 10^{-6}$ & $3.19_{-0.22}^{+0.22} \times 10^{36}$ \\
\hline \multicolumn{7}{|l|}{ Mature/old PWNe } \\
\hline HES & $5.67_{-1.15}^{+1.14} \times 10^{30}$ & $7.18_{-3.37}^{+2.23} \times 10^{33}$ & $8.16_{-1.33}^{+1.69} \times 10^{33}$ & $8.80_{-1.54}^{+1.36} \times 10^{34}$ & $8.96_{-2.43}^{+5.04} \times 10^{-4}$ & $3.10_{-1.21}^{+0.87} \times 10^{33}$ \\
\hline CTA 1 & $2.22_{-0.17}^{+2.94} \times 10^{29}$ & $4.97_{-2.55}^{+8.26} \times 10^{33}$ & $1.31_{-0.21}^{+1.57} \times 10^{33}$ & $1.85_{-0.64}^{+2.98} \times 10^{34}$ & $2.97_{-0.27}^{+0.27} \times 10^{-4}$ & $1.42_{-0.33}^{+1.81} \times 10^{33}$ \\
\hline HESS J1418-609 & $9.85_{-5.73}^{+14.11} \times 10^{30}$ & $4.49_{-4.32}^{+4.05} \times 10^{34}$ & $3.12_{-1.37}^{+2.16} \times 10^{34}$ & $3.33_{-1.92}^{+2.45} \times 10^{35}$ & $5.61_{-3.86}^{+3.33} \times 10^{-4}$ & $1.69_{-1.44}^{+1.37} \times 10^{34}$ \\
\hline HESS J1420-607 & $2.35_{-0.93}^{+1.56} \times 10^{31}$ & $4.15_{-2.25}^{+3.16} \times 10^{34}$ & $4.84_{-1.45}^{+2.18} \times 10^{34}$ & $4.68_{-1.53}^{+2.19} \times 10^{35}$ & $8.08_{-2.06}^{+3.32} \times 10^{-4}$ & $1.79_{-0.87}^{+1.27} \times 10^{34}$ \\
\hline HESS J1119-614 & $9.76_{-0.84}^{+0.82} \times 10^{31}$ & $1.30_{-0.36}^{+0.71} \times 10^{32}$ & $1.79_{-0.49}^{+0.67} \times 10^{34}$ & $3.72_{-0.29}^{+0.36} \times 10^{35}$ & $1.55_{-0.22}^{+0.29} \times 10^{-2}$ & $3.38_{-3.26}^{+3.61} \times 10^{28}$ \\
\hline HESS J1303-631 & $1.00_{-0.08}^{+0.08} \times 10^{30}$ & $1.92_{-0.02}^{+0.01} \times 10^{33}$ & $1.26_{-0.06}^{+0.05} \times 10^{35}$ & $4.26_{-0.25}^{+0.22} \times 10^{35}$ & $4.17 \times 10^{-3}$ & $5.09_{-0.05}^{+0.05} \times 10^{32}$ \\
\hline
\end{tabular}

young PWNe, $1<F_{\mathrm{X}} / F_{\gamma} \leq 10$ for evolved PWNe except for G292.0+1.8 $\left(F_{\mathrm{X}} / F_{\gamma} \sim 20.6\right)$, and $F_{\mathrm{X}} / F_{\gamma} \leq 1$ for mature/old PWNe except for CTA $1\left(F_{\mathrm{X}} / F_{\gamma} \sim 5\right)$. The results indicate that $F_{\mathrm{X}} / F_{\gamma}$ decreases with increasing PWN age.

\section{Correlation analysis of the PWNe}

In this section, the correlations of various physical quantities in our model are studied. To this end, the best linear fit $y=p_{1} x+p_{0}$ to the data with the minimum $\chi^{2}$ technique is used. For each fit, the values of $p_{1}$ and $p_{0}$ are given, and the Pearson's correlation coefficient $r$ and the probability of the null hypothesis $P_{\text {null }}$ are calculated. Here, it is assumed that the correlation between two physical quantities is noneffective if $P_{\text {null }} \geq 0.05$. In correlation analysis, the sample of 14 PWNe is used in which $\alpha_{2}, \eta_{\mathrm{B}}$, and $\epsilon$ are obtained through the LM method. All results have been recalculated and various luminosities with uncertainties are listed in Table 6. The uncertainties of the various physical quantities are included. We note that although four PWNe (N 158A, G310.61.6, G292.0+1.8, and HESS J1303-631) are not included in the best linear fits, the values of relevant physical quantities of these four PWNe are listed in Tables 4-6 and shown in Figs. 4-10.
Here, the $2 \sigma$ confidence band for each panel shown in Figs. 4-10 are from the dispersion of the fit to the points.

\subsection{Correlations between pulsar parameters with injection spectrum parameters and with derived parameters}

For a given central pulsar with a period $P(\mathrm{~s})$ and a period derivative $\dot{P}\left(\mathrm{~s} \mathrm{~s}^{-1}\right)$ in a PWN, it is commonly assumed that the magnetic field around it can be approximated as a dipole, so the surface magnetic field $B_{\mathrm{s}}$ is given by

$B_{\mathrm{S}}=\left(\frac{3 c^{3} I P \dot{P}}{2 \pi^{2} R^{6}}\right)^{1 / 2} \approx 6.4 \times 10^{19}(P \dot{P})^{1 / 2} \mathrm{G}$

where $c$ is the light speed, $I=10^{45} \mathrm{~g} \mathrm{~cm}^{2}$ is the inertia moment, and $R=10^{6} \mathrm{~cm}$ is the surface radius of the pulsar. At the light cylinder with a radius $R_{\mathrm{LC}}=(c P) /(2 \pi) \approx 4.77 \times 10^{9} P \mathrm{~cm}$, the magnetic field is described as

$B_{\mathrm{LC}}=B_{\mathrm{s}}\left(R / R_{\mathrm{LC}}\right)^{3} \approx 5.9 \times 10^{8} P^{-2.5} \dot{P}^{1 / 2} \mathrm{G}$. 
B.-T. Zhu et al.: Multiband nonthermal radiative properties of pulsar wind nebulae
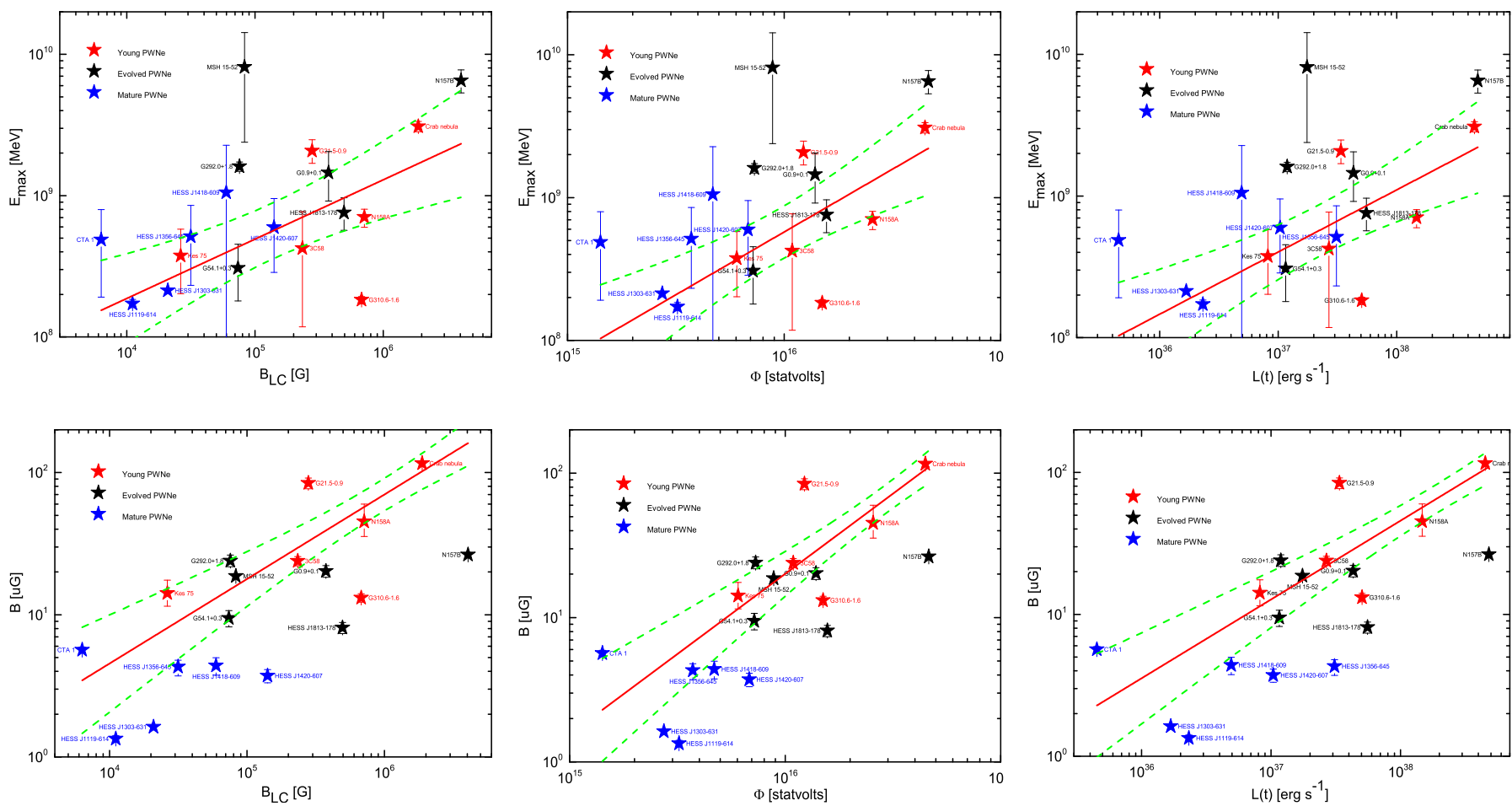

Fig. 4. Correlations between $E_{\max }$ (upper panels) and $B_{\mathrm{pwn}}$ (bottom panels) and pulsar parameters $B_{\mathrm{LC}}, \Phi$, and $L(t)$. The solid lines represent the best linear fits (from top left to bottom right): $\log E_{\max }=(0.42 \pm 0.10) \log B_{\mathrm{LC}}+(6.59 \pm 0.54), \log E_{\max }=(0.88 \pm 0.19) \log \Phi-(5.31 \pm 2.97)$, $\log E_{\max }=(0.44 \pm 0.09) \log L(t)-(7.65 \pm 3.46), \log B_{\mathrm{pwn}}=(0.59 \pm 0.08) \log B_{\mathrm{LC}}-(1.72 \pm 0.47), \log B_{\mathrm{pwn}}=(1.11 \pm 0.12) \log \Phi-(16.47 \pm 2.04)$, and $\log B_{\mathrm{pwn}}=(0.56 \pm 0.06) \log L(t)-(19.44 \pm 2.42)$; the correlation coefficient $r=0.71,0.76,0.76,0.88,0.91$, and 0.91 ; and the probability of the null hypothesis $P_{\text {null }}=8.95 \times 10^{-4}, 2.26 \times 10^{-4}, 2.24 \times 10^{-4}, 1.14 \times 10^{-6}, 1.23 \times 10^{-7}$, and $1.71 \times 10^{-7}$. The dashed lines are the $2 \sigma$ confidence bands for the sample.
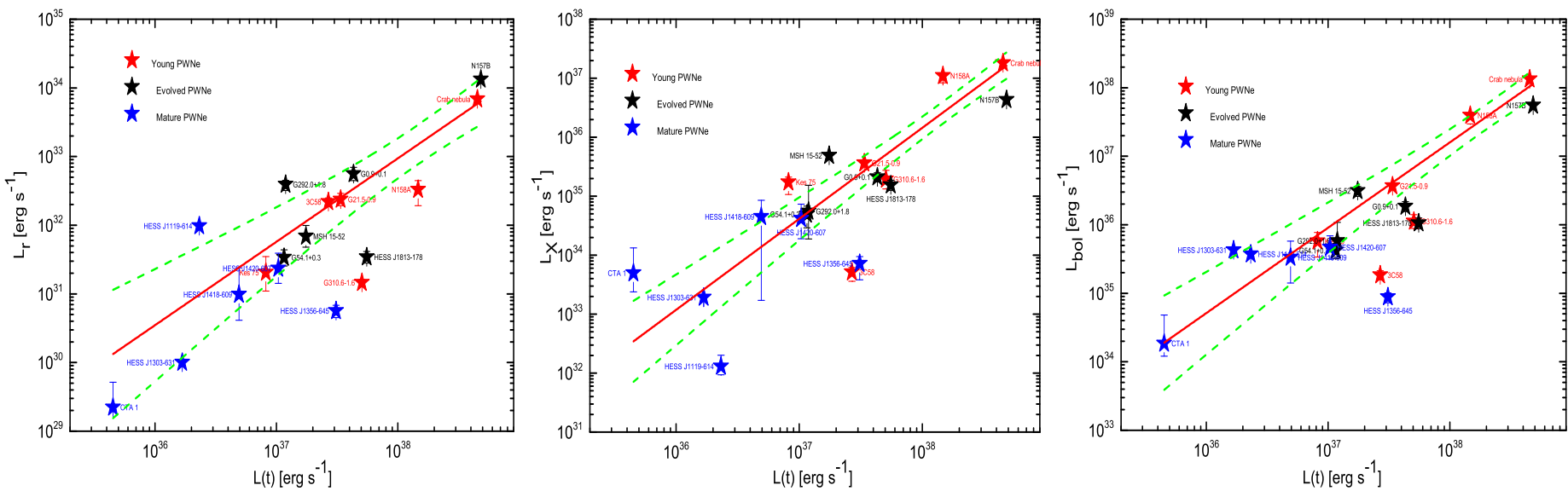

Fig. 5. Correlations between $L(t)$ and $L_{\mathrm{r}}, L_{\mathrm{X}}$, and $L_{\mathrm{bol}}$. The solid lines represent the best linear fits (from left to right): $\log L_{\mathrm{r}}=(1.21 \pm 0.17) \log L(t)-$ $(13.20 \pm 6.38), \log L_{\mathrm{X}}=(1.54 \pm 0.12) \log L(t)-(22.50 \pm 4.68)$, and $\log L_{\mathrm{bol}}=(1.24 \pm 0.12) \log L(t)-(10.15 \pm 4.57)$; the correlation coefficients $r=0.88,0.95$, and 0.93 ; and the probability of the null hypothesis $P_{\text {null }}=1.87 \times 10^{-6}, 1.03 \times 10^{-9}$, and $1.52 \times 10^{-8}$. The dashed lines are the $2 \sigma$ confidence bands for the sample.

For such a pulsar, the potential difference at the polar cap, $\Phi$, can be expressed as

$\Phi=\frac{2 \pi^{2} B_{\mathrm{s}} R^{3}}{(c P)^{2}}=4.2 \times 10^{20}\left(P^{-3} \dot{P}\right)^{1 / 2}$ stat volts

We note that the spin-down power $L(t)$ at time $t$ (see Eq. (3)) can be also expressed in terms of $P$ and $\dot{P}$ as

$L(t)=4 \pi^{2} I P^{-3} \dot{P} \approx 4.0 \times 10^{46} P^{-3} \dot{P} \operatorname{erg~s}^{-1}$.
For each pulsar, the $L(t)$ and $\tau_{\mathrm{c}}$ are shown in Tables $1-3$, and $B_{\mathrm{s}}$, $B_{\mathrm{LC}}$, and $\Phi$ are listed in Table 5.

The correlation analysis indicate that (1) there are no correlations between $E_{\mathrm{b}}, \eta_{\mathrm{B}}, \alpha_{1}, \alpha_{2}, \epsilon$ and the pulsar parameters such as $B_{\mathrm{s}}, B_{\mathrm{BL}}, \Phi, L(t)$, and $\tau_{\mathrm{c}}$; (2) two derived parameters $E_{\max }$ and $B_{\text {pwn }}$ have positive correlations with $B_{\mathrm{BL}}, \Phi$, and $L(t)$; and (3) there are no correlations between $B_{\mathrm{s}}$ and $E_{\max }$ and $B_{\mathrm{pwn}}$. The correlations between $E_{\max }$ and $B_{\mathrm{BL}}, \Phi$, and $L(t)$ are shown in the upper panel of Fig. 4 , and the correlations between $B_{\text {pwn }}$ and $B_{\mathrm{BL}}, \Phi$, and $L(t)$ in the bottom panel. Since $E_{\max } \sim L(t)^{1 / 2}$, 

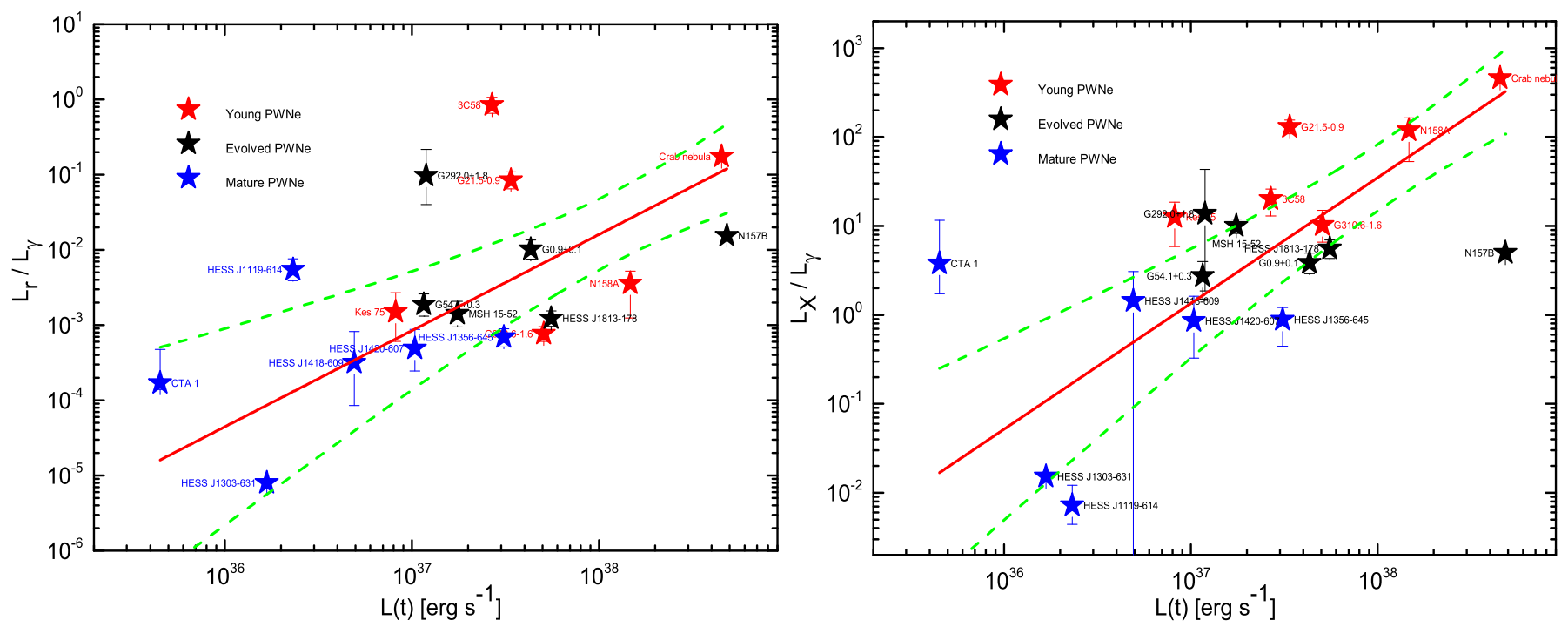

Fig. 6. Correlations between $L(t)$ and $L_{r} / L_{\gamma}$ and $L_{\mathrm{X}} / L_{\gamma}$. The solid lines represent the best linear fits (from left to right): $\log L_{\mathrm{r}} / L_{\gamma}=(1.28 \pm$ $0.28) \log L(t)-(50.38 \pm 10.53)$ and $\log L_{X} / L_{\gamma}=(1.41 \pm 0.22) \log L(t)-(50.21 \pm 8.33)$; the correlation coefficients $r=0.76$, and 0.85 ; and the probability of the null hypothesis $P_{\text {null }}=2.81 \times 10^{-4}$ and $7.81 \times 10^{-6}$. The dashed lines are the $2 \sigma$ confidence bands for the sample.
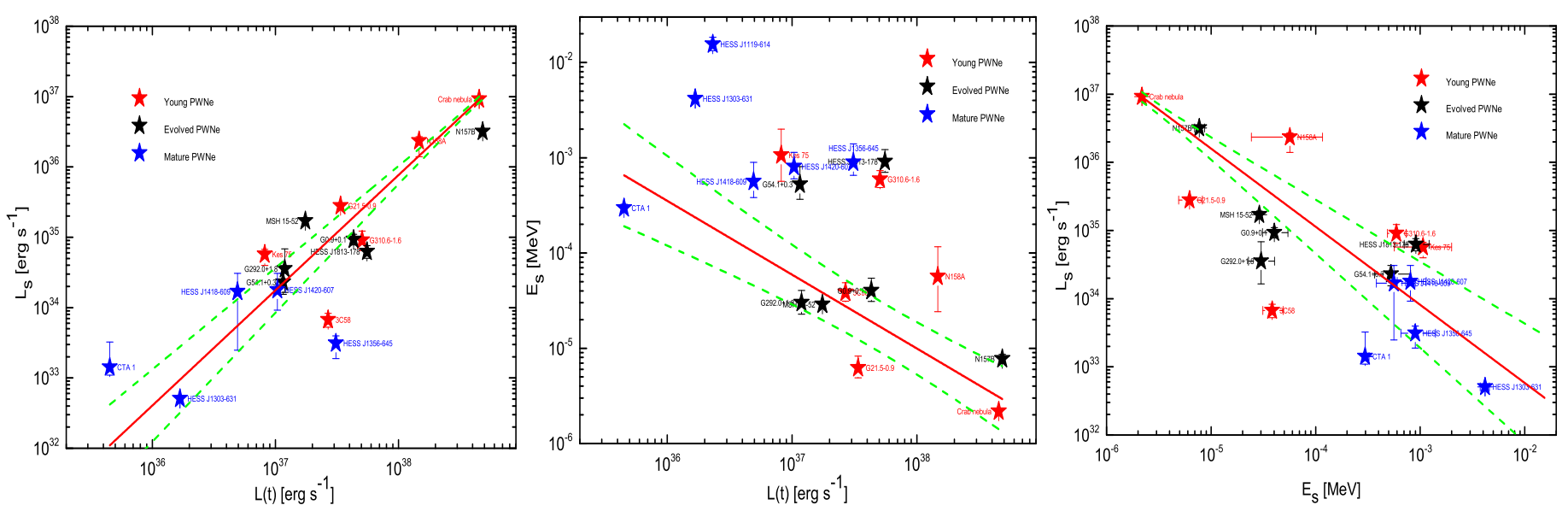

Fig. 7. Correlation between $L_{\mathrm{s}}, E_{\mathrm{s}}$ and $L(\mathrm{t}), L_{\mathrm{s}}$, and $E_{\mathrm{s}}$. The solid lines are the best linear fits, which $\operatorname{are} \log L_{\mathrm{s}}=(1.64 \pm 0.09) \log L(t)-(26.39 \pm 3.55)$, $\log E_{\mathrm{s}}=-(0.78 \pm 0.11) \log L(t)+(24.46 \pm 3.99)$, and $\log L_{\mathrm{s}}=-(1.15 \pm 0.11) \log E_{\mathrm{s}}+(30.47 \pm 0.64)$; the correlation coefficients $r=0.98,0.88$, and 0.93 ; and the probability of the null hypothesis $P_{\text {null }}=5.59 \times 10^{-12}, 1.70 \times 10^{-6}$, and $2.27 \times 10^{-8}$ (from left to right). The dashed lines are the $2 \sigma$ confidence bands for the sample.

$L(t) \sim(P \dot{P})^{1 / 2}, B_{\mathrm{LC}} \sim P^{5 / 2} \dot{P}^{1 / 2}$, and $\Phi \sim\left(P^{-3} \dot{P}\right)^{1 / 2}$, these correlations are only consistent with the logical conclusions.

\subsection{Correlations between luminosities at various bands and $L(t)$}

The possible correlations between the luminosities at radio $(1.4 \mathrm{GHz}), L_{\mathrm{r}}$; X-ray $(1-10 \mathrm{keV}), L_{\mathrm{X}}$; VHE $\gamma$-ray $(1-10 \mathrm{TeV})$, $L_{\gamma}$; bolometric luminosity at all wave bands, $L_{\text {bol }}$ (which can be obtained from our model results, the corresponding values and errors are listed in Table 5); and the pulsar's spin-down power, $L(t)$, were analyzed. The results show that there are significant positive correlations between $L_{\mathrm{r}}$ and $L(t)$, between $L_{\mathrm{X}}$ and $L(t)$, and between $L_{\mathrm{bol}}$ and $L(t)$ (see Fig. 5), and the best linear fits yield $L_{\mathrm{r}} \sim L(t)^{1.21 \pm 0.17}, L_{\mathrm{X}} \sim L(t)^{1.54 \pm 0.12}$, and $L_{\mathrm{bol}} \sim L(t)^{1.24 \pm 0.12}$, respectively. The correlation between $L_{\mathrm{X}}$ and $L(t)$ is in agreement with the recent conclusions of Mattana et al. (2009), Kargaltsev \& Pavlov (2010), and Kargaltsev et al. (2013). As expected, there is no correlation between $L_{\gamma}$ and $L(t)$, which is consistent with Mattana et al. (2009), Kargaltsev \& Pavlov (2010), and Kargaltsev et al. (2013). This phenomenon may be due to the differences in the IR background and uncertain distance.

Since $L_{r} / L_{\gamma}$ and $L_{X} / L_{\gamma}$ are distance-independent, the correlations between $L_{r} / L_{\gamma}$ and $L(t)$ and between $L_{X} / L_{\gamma}$ and $L(t)$ are analyzed. Our results are shown in Fig. 6 where the best linear fits give $L_{R} / L_{\gamma} \sim L(t)^{1.28 \pm 0.28}$, and $L_{\mathrm{X}} / L_{\gamma} \sim L(t)^{1.41 \pm 0.22}$. In fact, the correlation between $L_{\mathrm{X}} / L_{\gamma}$ and $\mathrm{L}(\mathrm{t})$ is found in Mattana et al. (2009).

\subsection{Correlations between synchrotron luminosity and $L(t)$}

Because synchrotron radiation dominates over the radiation from radio to X-ray bands, it is important to study the correlations of synchrotron cooling break energy $\left(E_{\mathrm{s}}\right)$ and synchrotron radiation luminosity $\left(L_{\mathrm{s}}\right)$ with $L(t)$. Following Tanaka \& Takahara (2011), 

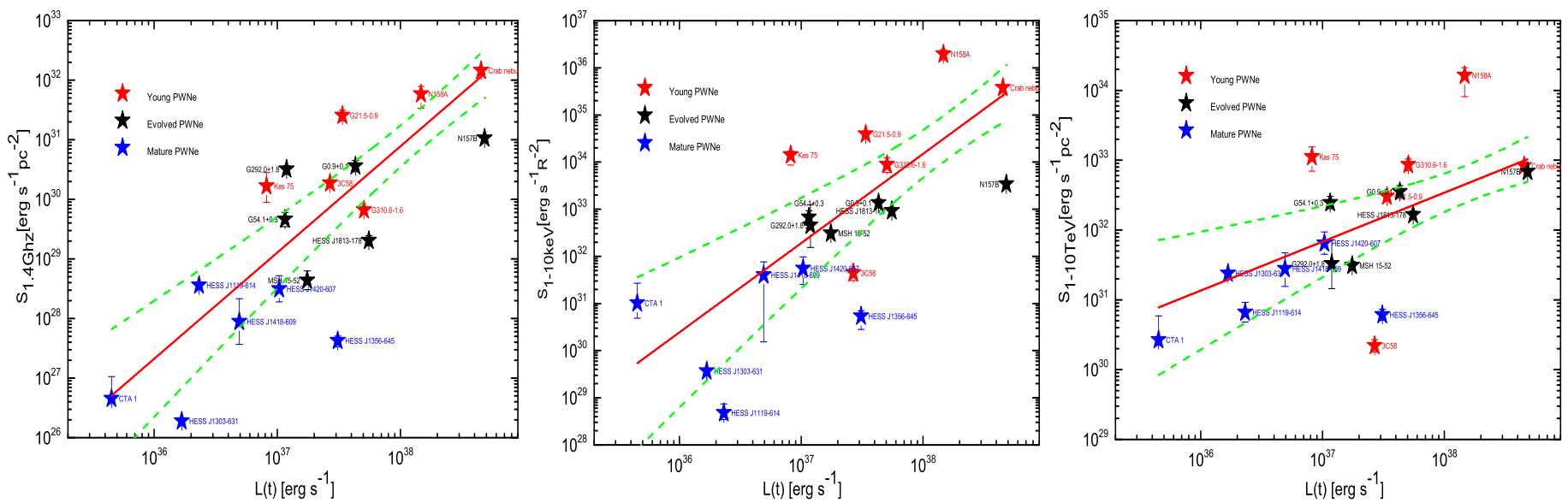

Fig. 8. Correlations between $L(t)$ and $S_{\mathrm{r}}, S_{\mathrm{X}}$, and $S_{\gamma}$. The solid lines represent the best linear fits (from left to right): $\log S_{\mathrm{r}}=(1.78 \pm 0.20) \log L(t)-$ (36.89 \pm 7.60$), \log S_{\mathrm{X}}=(1.89 \pm 0.32) \log L(t)-(37.79 \pm 12.42)$, and $\log S_{\gamma}=(0.71 \pm 0.17) \log L(t)+(5.89 \pm 6.59)$; the correlation coefficients $r=0.91,0.82$, and 0.71 ; and the probability of the null hypothesis $P_{\text {null }}=1.24 \times 10^{-7}, 2.57 \times 10^{-5}$, and $9.05 \times 10^{-4}$, respectively. The dashed lines are the $2 \sigma$ confidence bands for the sample.
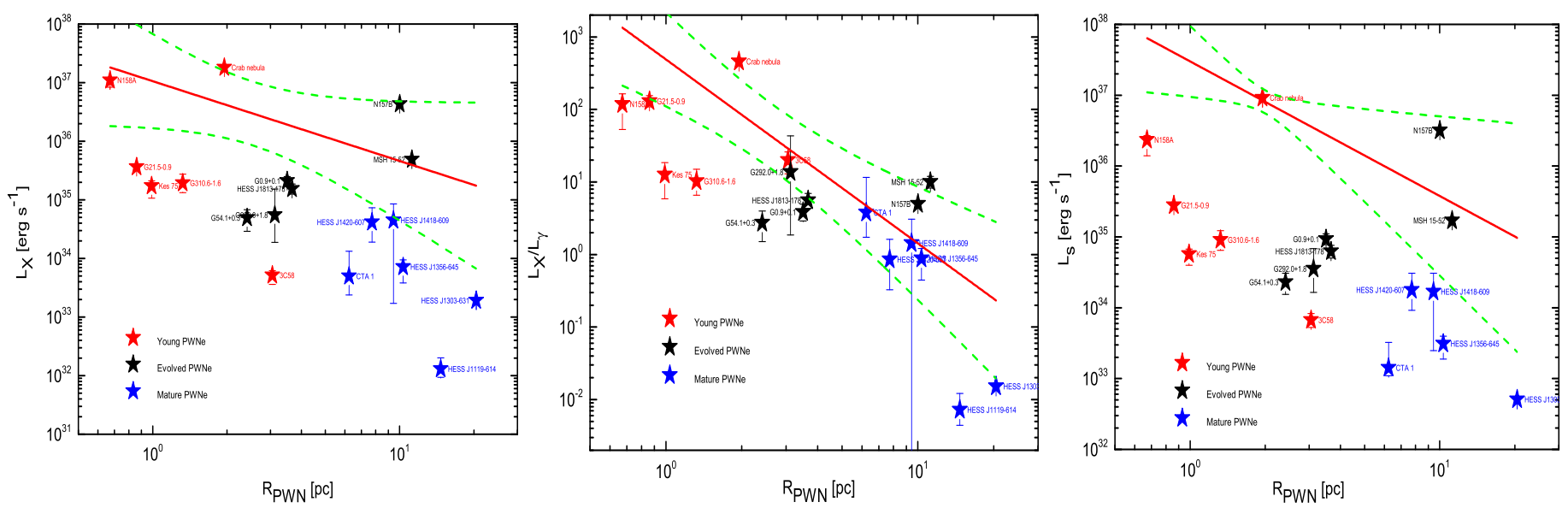

Fig. 9. Correlations between $R_{\mathrm{pwn}}$ and $L_{\mathrm{X}}, L_{\mathrm{X}} / L_{\gamma}$, and $L_{\mathrm{s}}$. The solid lines are the best linear fits, which $\operatorname{are} \log L_{\mathrm{X}}=-(1.36 \pm 0.68) \log R_{\mathrm{pwn}}+$ $(37.03 \pm 0.38), \log L_{\mathrm{X}} / L_{\gamma}=-(2.54 \pm 0.52) \log R_{\mathrm{pwn}}+(2.69 \pm 0.31)$, and $\log L_{\mathrm{s}}=-(1.90 \pm 0.75) \log R_{\mathrm{PWNe}}+(37.48 \pm 0.24)$; the correlation coefficients $r=0.45,0.77$, and 0.54 ; and the probability of the null hypothesis $P_{\text {null }}=6.40 \times 10^{-2}, 1.73 \times 10^{-4}$, and $2.18 \times 10^{-2}$ (from left to right). The dashed lines are the $2 \sigma$ confidence bands for the sample.

the inverse Compton cooling is assumed to be ineffective in most of the evolutionary phases, and the synchrotron cooling break frequency $v_{s}$ is given by

$v_{\mathrm{s}}=7.32 \times 10^{20}\left(\frac{B_{\mathrm{PWN}}}{10^{-6} \mathrm{G}}\right)^{-3}\left(\frac{T_{\mathrm{age}}}{10^{3} \mathrm{yr}}\right)^{-2} \mathrm{~Hz}$.

Using Eq. (13) and based on our model calculation, $E_{\mathrm{s}}=h v_{\mathrm{s}}$ and $L_{\mathrm{s}}$ are calculated for each PWN in our sample. The results show that there is a strong positive correlation between $L_{\mathrm{s}}$ and $L(t)$, the best linear fit yields $L_{\mathrm{s}} \sim L(t)^{1.64 \pm 0.09}$, and that there are strong negative correlation between $E_{\mathrm{s}}$ and $\mathrm{L}(\mathrm{t})$, where $E_{\mathrm{s}} \sim$ $L(t)^{-0.78 \pm 0.11}$, and $E_{\mathrm{s}}$ and $L_{\mathrm{s}}$, where $L_{\mathrm{s}} \sim E_{\mathrm{s}}^{-1.15 \pm 0.11}$. The best linear fit results are shown in Fig. 7.

\subsection{Correlations between surface brightness $S$ and $L(t)$}

The surface brightness is defined as (e.g., Abdalla et al. 2017)

$S=\frac{L}{4 \pi R_{\mathrm{pwn}}^{2}} \approx \frac{F}{\sigma^{2}}$, where $L$ is the luminosity at a given band, $R_{\mathrm{PWN}}$ is the radius of the PWN, $F$ is the energy flux at a given band, and $\sigma$ is its angular extent as seen from the Earth. Using our model and Eq. (14), the surface brightness, $S_{\mathrm{R}}, S_{\mathrm{X}}$, and $S_{\gamma}$, at radio $(1.4 \mathrm{GHz}), \mathrm{X}$-ray (1-10 keV), and $\gamma$-ray (1-10 TeV) bands are calculated. The results are shown in Fig. 8 and show that there are correlations between $\mathrm{L}(\mathrm{t})$ and $S_{\mathrm{R}}, S_{\mathrm{X}}$, and $S_{\gamma}$. The best linear fits give $S_{\mathrm{r}} \sim L(t)^{1.78 \pm 0.20}, S_{\mathrm{X}} \sim L(t)^{1.89 \pm 0.32}$, and $S_{\gamma} \sim L(t)^{0.71 \pm 0.17}$. We note that $S_{\gamma} \sim L(t)^{0.81}$ has been given in Abdalla et al. (2017).

\subsection{Correlations between luminosity and $R_{\text {pwn }}$ and $T_{\text {age }}$}

The correlations between the luminosities at various bands and $R_{\mathrm{pwn}}$ are analyzed here. In our model, the PWN radius for each PWN can be calculated (see Tables $1-3$ ). The results show that there are no correlations between $R_{\mathrm{pwn}}$ and $L_{\mathrm{r}}, L_{\gamma}$, and $L_{\mathrm{bol}}$, but there are correlations between $R_{\mathrm{pwn}}$ and $L_{\mathrm{X}}, L_{\mathrm{s}}$, and $L_{\mathrm{X}} / L_{\gamma}$. The best linear fits give that $L_{\mathrm{X}} \sim R_{\mathrm{pwn}}^{-1.36 \pm 0.68}, L_{\mathrm{s}} \sim R_{\mathrm{pwn}}^{-1.90 \pm 0.75}$, and $L_{\mathrm{X}} / L_{\gamma} \sim R_{\mathrm{pwn}}^{-2.54 \pm 0.52}$, respectively. We note that the correlation 

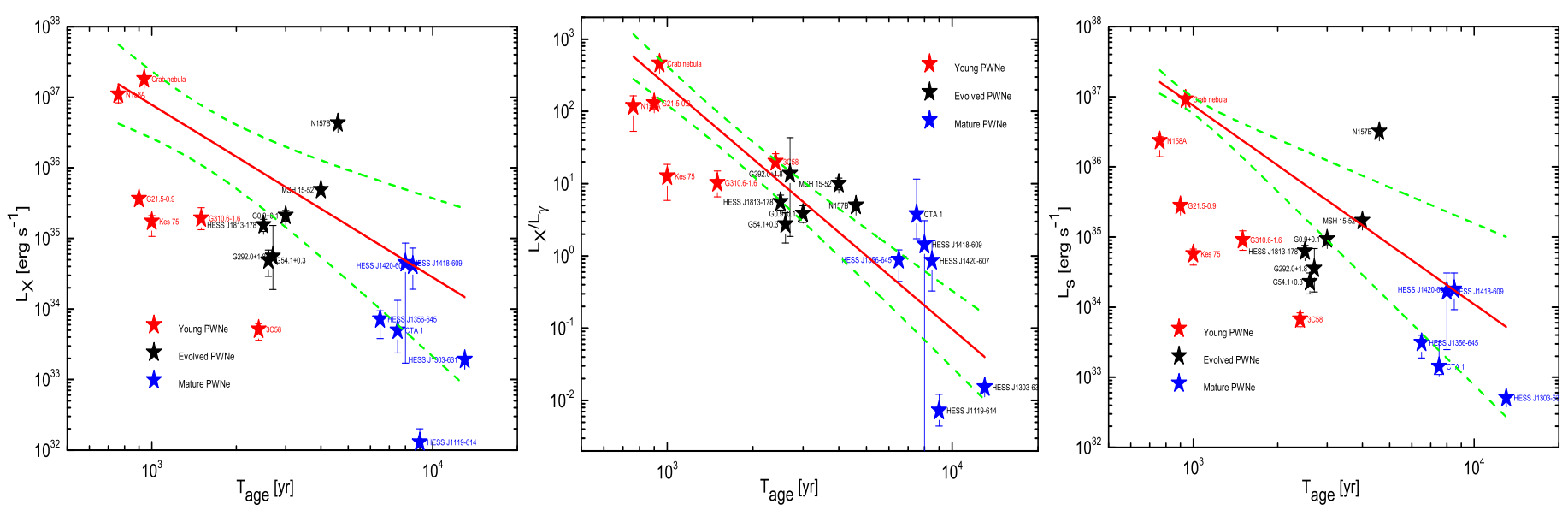

Fig. 10. Correlations between $T_{\text {age }}$ and $L_{\mathrm{X}}, L_{\mathrm{X}} / L_{\gamma}$, and $L_{\mathrm{s}}$. The solid lines are the best linear fits, which $\operatorname{are} \log L_{\mathrm{X}}=-(2.44 \pm 0.59) \log T_{\mathrm{age}}+$ (44.23 \pm 1.89$), \log L_{\mathrm{X}} / L_{\gamma}=-(3.37 \pm 0.29) \log T_{\text {age }}+(12.47 \pm 0.94)$, and $\log L_{\mathrm{s}}=-(2.83 \pm 0.53) \log T_{\text {age }}+(45.37 \pm 1.59)$; the correlation coefficients $r=0.72,0.95$, and 0.80 ; and the probability of the null hypothesis $P_{\text {null }}=7.81 \times 10^{-4}, 3.15 \times 10^{-9}$, and $7.22 \times 10^{-5}$. The dashed lines are the $2 \sigma$ confidence bands for the sample.

between $L_{\mathrm{X}}$ and $R_{\mathrm{pwn}}$ is marginal here. The results are shown in Fig. 9.

The same procedure is performed for the correlations between $T_{\text {age }}$ and the luminosities at various bands, and almost the same correlations are found. We found that there are correlations between $T_{\text {age }}$ and $L_{\mathrm{X}}, L_{\mathrm{s}}$, and $L_{\mathrm{X}} / L_{\gamma}$. These correlations mean that $L_{\mathrm{X}} \sim T_{\mathrm{age}}^{-2.44 \pm 0.59}, L_{\mathrm{S}} \sim T_{\mathrm{age}}^{-2.83 \pm 0.53}$, and $L_{\mathrm{X}} / L_{\gamma} \sim T_{\mathrm{age}}^{-3.37 \pm 0.29}$. The best linear fit results are shown in Fig. 10.

\section{Discussions and conclusions}

In this paper, the leptonic model with a broken power-law injection for the electrons/positrons is applied to the sample of 18 PWNe. The sample is divided into three groups: young, evolved, and mature/old PWNe. Observed SEDs of all 18 PWNe can be reproduced well in this model (see Figs. 1-3). The model parameters obtained in our calculations are listed in the Tables 1-3, and the relevant discussion are described in Sect. 3.

Using a time-dependent modeling of PWNe given by Martin et al. (2012), Torres et al. (2014) modeled the SEDs of nine PWNe and studied their statistical properties. These nine PWNe are included in our sample. As mentioned in Sect. 1, the model given in Torres et al. (2014) does not include the dynamical evolution of a PWN, but our model does. Therefore, some model parameters obtained in these two models are different. Meanwhile, our sample is larger than some previous works. So it is worth comparing our correlation results with the results of Torres et al. (2014) and some previous works.

For the relation between the derived parameters and pulsar's parameter, our results indicate that the maximum electron energy has positive correlations with the magnetic field at the light cylinder, the potential difference at the polar cap, and the pulsar's spin-down power and that the magnetic field in the PWN is positively correlated with the magnetic field at the light cylinder, the potential difference at the polar cap, and the pulsar's spin-down power (see Fig. 4). Other parameters that describe pulsar properties and electron injection have no correlations. These results are consistent with those of Torres et al. (2014).

The results presented in this paper indicate that the spindown power $L(t)$ is correlated with $L_{\mathrm{r}}, L_{\mathrm{X}}$, and $L_{\mathrm{bol}}$ (see Fig. 5). The results of $L(t)$ versus $L_{\mathrm{r}}$ and $L(t)$ versus $L_{\mathrm{X}}$ are consistent with those in Torres et al. (2014). Meanwhile, our results show that the $L(t)$ correlates with the ratio $L_{\mathrm{r}} / L_{\gamma}$ and the ratio $L_{\mathrm{X}} / L_{\gamma}$ (see Fig. 6). These correlations are consistent with those given in previous papers (e.g., Mattana et al. 2009; Kargaltsev \& Pavlov 2010; Torres et al. 2014).

In this paper, the correlations between $L(t)$ and surface brightness at different bands are presented here (see Fig. 8). Moreover, the correlations between $R_{\mathrm{pwn}}\left(T_{\text {age }}\right)$ and $L_{\mathrm{X}}, L_{\mathrm{X}} / L_{\gamma}$, and $L_{\mathrm{s}}$ are analyzed (see Figs. 9 and 10). For example, the results show that $L_{\mathrm{X}} / L_{\gamma} \sim R_{\mathrm{pwn}}^{-2.54}$ and $L_{\mathrm{X}} / L_{\gamma} \sim T_{\mathrm{age}}^{-3.37}$, which means that old PWNe have smaller values of $L_{\mathrm{X}} / L_{\gamma}$ than do young PWNe. In fact, our results show that the $L_{X} / L_{\gamma} \geq 10$ for young PWNe, $1<L_{\mathrm{X}} / L_{\gamma} \lesssim 10$ for evolved PWNe, and $L_{\mathrm{X}} / L_{\gamma} \leq 1$ for mature/old PWNe. This result may provide a new tool for classifying the evolution state of PWNe. It should be pointed out that the results presented in this paper require confirmation from further observations.

Acknowledgements. We would like to thank the anonymous referee for the very constructive comments. This work is partially supported by the National Natural Science Foundation of China (NSFC 11433004, 11173020, 11563009), the Doctoral Fund of the Ministry of Education of China (RFDP 20115301110005), and the Research Innovation Fund for Graduate Students of Yunnan University.

\section{References}

Abdalla, H., Abramowski, A., Aharonian, F., et al. 2017, A\&A, in press, DOI: $10.1051 / 0004-6361 / 201629377$

Abdo, A. A., Ackermann, M., Atwood, W. B., et al. 2008, Science, 322, 1218

Abdo, A. A., Ackermann, M., Ajello, M., et al. 2010, ApJ, 714, 927

Abdo, A. A., Wood, K. S., DeCesar, M. E., et al. 2012, ApJ, 744, 146 Abdo, A. A., Ajello, M., Allafort, A., et al. 2013, ApJS, 208, 17

Abramowski, A., Acero, F., Aharonian, F., et al. 2011, A\&A, 533, A103

Abramowski, A., Acero, F., Aharonian, F., et al. 2012a, A\&A, 545, L2

Abramowski, A., Acero, F., Aharonian, F., et al. 2012b, A\&A, 548, A46

Abramowski, A., Aharonian, F., Ait Benkhali, F., et al. 2015, Science, 347, 406 Acciari, V. A., Aliu, E., Arlen, T., et al. 2010, ApJ, 719, L69

Acero, F., Ackermann, M., Ajello, M., et al. 2013, ApJ, 773, 77

Achterberg, A., Gallant, Y. A., Kirk, J. G., \& Guthmann, A. W. 2001, MNRAS, 328,393

Aharonian, F., Akhperjanian, A. G., Aye, K.-M., et al. 2005a, A\&A, 435, L17 Aharonian, F., Akhperjanian, A. G., Aye, K.-M., et al. 2005b, A\&A, 432, L25 Aharonian, F., Akhperjanian, A. G., Aye, K.-M., et al. 2005c, A\&A, 439, 1013 Aharonian, F., Akhperjanian, A. G., Bazer-Bachi, A. R., et al. 2006a, A\&A, 456, 245

Aharonian, F., Akhperjanian, A. G., Bazer-Bachi, A. R., et al. 2006b, ApJ, 636, 777

Ackermann, M., Ajello, M., Baldini, L., et al. 2011, ApJ, 726, 35

Ackermann, M., Ajello, M., Allafort, A., et al. 2013, ApJS, 209, 34

Aleksić, J., Ansoldi, S., Antonelli, L. A., et al. 2014, A\&A, 567, L8

Aliu, E., Archambault, S., Arlen, T., et al. 2013, ApJ, 764, 38 
Archibald, R. F., Kaspi, V. M., Beardmore, A. P., Gehrels, N., \& Kennea, J. A. 2015, ApJ, 810, 67

Becker, R .H., \& Kundu, M. R. 1976, ApJ, 204, 427

Bhalerao, J., Park, S., Dewey, D., et al. 2015, ApJ, 800, 65

Bietenholz, M. F., \& Bartel, N. 2008, MNRAS, 386, 1411

Bock, D. C.-J., \& Gaensler, B. M. 2005, ApJ, 626, 343

Bocchino, F., Bandiera, R., \& Gelfand, J. 2010, A\&A, 520, A71

Brogan, C. L., Gaensler, B. M., Gelfand, J. D., et al. 2005, ApJ, 629, L105

Bucciantini, N. 2008, in 40 Years of Pulsars Millisecond Pulsars, Magnetars and More, eds. C. Bassa, Z. Wang, A. Cumming, \& V. M. Kaspi, AIP Conf. Ser. 983, 186

Bucciantini, N., Arons, J., \& Amato, E. 2011, MNRAS, 410, 381

Bühler, R., \& Blandford, R. 2014, Rept. Progr. Phys., 77, 06690

Camilo, F., Kaspi, V. M., Lyne, A. G., et al. 2000, ApJ, 541, 367

Camilo, F., Manchester, R. N., Gaensler, B. M., Lorimer, D. R., \& Sarkissian, J. 2002a, ApJ, 567, L71

Camilo, F., Stairs, I. H., Lorimer, D. R., et al. 2002b, ApJ, 571, L41

Camilo, F., Manchester, R. N., Lyne, A. G., et al. 2004, ApJ, 611, L25

Camilo, F., Ransom, S. M., Gaensler, B. M., et al. 2006, ApJ, 637, 456

Camilo, F., Ransom, S. M., Gaensler, B. M., \& Lorimer, D. R. 2009, ApJ, 700, L34

Campana, R., Mineo, T., De Rosa, A., et al. 2008, MNRAS, 389, 691

Caswell, J. L., Milne, D. K., \& Wellington, K. J. 1981, MNRAS, 195, 89

Caswell, J. L., McClure-Griffiths, N. M., \& Cheung, M. C. M. 2004, MNRAS 352,1405

Chang, C., Pavlov, G. G., Kargaltsev, O., \& Shibanov, Yu. A. 2012, ApJ, 744, 81

Chevalier, R. A. 2004, Adv. Space Res., 33, 456

Chevalier, R. A. 2005, ApJ, 619, 839

Chen, Y., Wang, Q. D., Gotthelf, E. V., et al. 2006, ApJ, 651, 237

Condon, J. J., Griffith, M. R., \& Wright, A. E. 1993, AJ, 106, 1095

Cordes, J. M., \& Lazio, T. J. W. 2002, ArXiv e-prints [arXiv:astro-ph/0207156]

Crawford, F., Gaensler, B. M., Kaspi, V. M., et al. 2001, ApJ, 554, 152

D’Amico, N., Kaspi, V. M., Manchester, R. N., et al. 2001, ApJ, 552, L45

De Rosa, A., Ubertini, P., Campana, R., et al. 2009, MNRAS, 393, 527

Djannati-Atai, A., deJager, O. C., Terrier, R., Gallant, Y. A., \& Hoppe, S. 2008, Proc. 30th International Cosmic Ray Conference, eds. R. Caballero et al., 2 823

Dubner, G., Giacani, E., \& Decourchelle, A. 2008, A\&A, 487, 1033

Duncan, A. R., Stewart, R. T., Haynes, R. F., \& Jones, K. L. 1995, MNRAS, 277,

Fang, J., \& Zhang, L. 2010a, A\&A, 515, A20

Fang, J., \& Zhang, L. 2010b, ApJ, 718, 467

Ferdman, R. D., Archibald, R. F., \& Kaspi, V. M. 2015, ApJ, 812, 95

Forot, M., Hermsen, W., Renaud, M., et al. 2006, ApJ, 651, L45

Funk, S., Hinton, J. A., Moriguchi, Y., et al. 2007, A\&A, 470, 249

Gaensler, B. M., \& Slane, P. O. 2006, ARA\&A, 44, 17

Gaensler, B. M., \& Wallace, B. J. 2003, ApJ, 594, 326

Gaensler, B. M., Brazier, K. T. S., Manchester, R. N., Johnston, S., \& Green, A. J. 1999, MNRAS, 305, 724

Gaensler, B. M., Arons, J., Kaspi, V. M., et al. 2002, ApJ, 569, 878

Gaensler, B. M., Arons, J., Kaspi, V. M., et al. 2008, ApJ, 569, 878

Gallant, Y. A., \& Tuffs, R. J. 1998, Mem. Soc. Astron. It., 69, 963

Gallant, Y. A., \& Tuffs, R. J. 1999, ESASP, 427, 313

Gelfand, J. D., Slane, P. O., \& Zhang, W. 2009, ApJ, 703, 2051

Gelfand, J. D., Slane, P. O., \& Temim, T. 2014, Astron. Nachr., 335, 318

Gelfand, J. D., Slane, P. O., \& Temim, T. 2015, ApJ, 807, 30

Gonzalez, M., \& Safi-Harb, S. 2003, ApJ, 591, L143

Gotthelf, E. V., \& Halpern, J. P. 2009, ApJ, 700, L158

Gotthelf, E. V., Vasisht, G., Boylan-Kolchin, M., \& Torii, K. 2000, ApJ, 542, L37

Green, D. A. 1985, MNRAS, 216, 691

Green, D. A. 1986, MNRAS, 218, 533

Green, D. A. 1994, ApJS, 90, 817

Griffith, M. R., \& Wright, A. E. 1993, AJ, 105, 1666

Gupta, Y., Mitra, D., Green, D. A., \& Acharyya, A. 2005, CSci, 89, 853

Goldreich, P., \& Julian, W. 1969, ApJ, 157, 869

Gvaramadze, V. V. 2001, A\&A, 374, 259

Halpern, J. P., Gotthelf, E. V., Camilo, F., Helfand, D. J., \& Ransom, S. M. 2004, ApJ, 612, 398

Halpern, J. P., Gotthelf, E. V., \& Camilo, F. 2012, ApJ, 753, L14

Harris, D. E., \& Roberts, J. A. 1960, PASP, 72, 347

Helfand, D. J., Collins, B. F., \& Gotthelf, E. V. 2003, ApJ, 582, 783

Hughes, J. P., Slane, P. O., Burrows, D. N., et al. 2001, ApJ, 559, L153

Indebetouw, R., de Messières, G. E., Madden, S., et al. 2009, ApJ, 694, 84

Kaaret, P., Marshall, H. L., Aldcroft, T. L., et al. 2001, ApJ, 546, 1159

Kargaltsev, O., \& Pavlov, G. G. 2010, in X-Ray Astronomy 2009; Present Status, Multi-wavelength Approach and Future Perspectives, eds. A. Comastri, M. Cappi, \& L. Angelini (Melville, NY: AIP), AIP Conf. Proc. 1248, 25
Kargaltsev, O., Rangelov, B., \& Pavlov, G. G. 2013, in The Universe Evolution. Astrophysical and Nuclear Aspects, eds. Strakovsky \& Blokhintsev (Nova Science Publishers, Inc)

Kennel, C. F., \& Coroniti, F. V. 1984, ApJ, 283, 694

Khélifi, B., Masterson, C., Pita, S., \& Oña-Wilhelmi, E. 2008, in New Companions for the Lonely Crab? VHE Emission from Young Pulsar Wind Nebulae Revealed by HESS Universidad Nacional Autónoma de México, Mexico City, eds. R. Caballero, J. Carlos D’Olivo, G. Medina-Tanco, et al., Proc. 30th Int. Cosm. Ray Conf., 2, 803

Kirshner, R. P., Morse, J. A., Winkler, P. F., \& Blair, W. P. 1989, ApJ, 342, 260

Kishishita, T., Bamba, A., Uchiyama, Y., Tanaka, Y., \& Takahashi, T. 2012, ApJ, 750,162

Kothes, R. 2010, in The Dynamic Interstellar Medium: A Celebration of the Canadian Galactic Plane Survey, eds. R. Kothes, T. L. Landecker, \& A. G. Willis (San Francisco: ASP), ASP Conf. Ser., 438, 347

Kothes, R. 2013, A\&A, 560, A18

Kumar, H. S., Safi-Harb, S., \& Gonzalez, M. E. 2012, ApJ, 754, 96

Lang, C. C., Wang, Q. D., Lu, F., \& Clubb, K. I. 2010, ApJ, 709, 1125

Lazendic, J. S., Dickel, J. R., Haynes, R. F., Jones, P. A., \& White, G. L. 2000 , ApJ, 540, 808

Leahy, D. A., \& Tian, W. W. 2008, ApJ, 480, L25

Leahy, D., Tian, W., \& Wang, Q. D. 2008, ApJ, 136, 1477

Livingstone, M. A., Kaspi, V. M., Gavril, F. P., \& Manchester, R. N. 2005a, ApJ, 619,1046

Livingstone, M. A., Kaspi, V. M., \& Gavriil, F. P. 2005b, ApJ, 633, 1095

Livingstone, M. A., Kaspi, V. M., Gotthelf, E. V., \& Kuiper, L. 2006, ApJ, 647, 1286

Livingstone, M. A., Ransom, S. M., Camilo, F., et al. 2009, ApJ, 706, 1163

Lorimer, D. R., Faulkner, A. J., Lyne, A. G., et al. 2006, MNRAS, 372, 777

Lu, F. J., Aschenbach, B., \& Song, L. M. 2001, A\&A, 370, 570

Lu, F. J., Wang, Q. D., Aschenbach, B., Durouchoux, P., \& Song, L. M. 2002, ApJ, 568, L49

Lu, F., Wang, Q. D., Gotthelf, E. V., et al. 2007, ApJ, 663, 315

Manchester, R. N., Staveley-Smith, L., \& Kesteven, M. J. 1993, ApJ, 411, 756

Manchester, R. N., Hobbs, G. B., Teoh, A., \& Hobbs, M. 2005a, ViZier On-line Data Catalog: VII/245

Manchester, R. N., Hobbs, G. B., Teoh, A., \& Hobbs, M. 2005b, AJ, 129, 1993

Macri, L. M., Stanek, K. Z., Bersier, D., Greenhill, L. J., \& Reid, M. J. 2006 , ApJ, 652, 1133

Mattana, F., Falanga, M., Götz, D., et al. 2009, ApJ, 694, 12

Martin, J., Torres D. F., \& Rea N. 2012, MNRAS, 427, 415

Martin, J., Torres, D. F., Cillis A., \& de Ona Wilhelmi, E. 2014, MNRAS, 443, 138

Martin J., Torres D. F., \& Pedaletti, G. 2016, MNRAS, 459, 3868

Marshall, F. E., Gotthelf, E. V., Zhang, W., Middleditch, J., \& Wang, Q. D. 1998, ApJ, 499, L179

Matheson, H., \& Safi-Harb, S. 2005, Adv. Space Res., 35, 1099

Mereghetti, S., Bandiera, R., Bocchino, F., \& Israel, G. L. 2002, ApJ, 574, 873

Mills, B. Y., Slee, O. B., \& Hill, E. R. 1961, Austr. J. Phys., 14, 497

Mignani, R. P., De Luca, A., Hummel, W., et al. 2012, A\&A, 544, A100

Mineo, T., Cusumano, G., Maccarone, M. C., et al. 2001, A\&A, 380, 695

Morsi, H. W., \& Reich, W. 1987, A\&AS, 69, 533

Murphy, T., Mauch, T., Green, A., et al. 2007, MNRAS, 382, 382

Murdin, P., \& Clark, D. H. 1979, MNRAS, 189, 501

Murray, S. S., Slane P. O., Seward F. D., Ransom S. M., \& Gaensler B. M. 2002, ApJ, 568, 226

Nynka, M. Hailey, C. J., Reynolds, S. P., et al. 2014, ApJ, 789, 72

Ng, C.-Y., Roberts, M. S. E., \& Romani, R. W. 2005, ApJ, 627, 904

Pineault, S., Landecker, T. L., Madore, B., \& Gaumont-Guay, S. 1993, AJ, 105, 1060

Porquet, D., Decourchelle, A., \& Warwick, R. S. 2003, A\&A, 401, 197

Porter, T. A., Moskalenko, I. V., \& Strong, A. W. 2006, ApJ, 648 , L29

Rees, M. J., \& Gunn, J. E. 1974, MNRAS, 167, 1

Reich, W., Fuerst, E., Altenhoff, W. J., Reich, P., \& Junkes, N. 1985, A\&A, 151, L10

Renaud, M., Marandon, V., Gotthelf, E. V., et al. 2010, ApJ, 716, 670

Reynolds, S. P. 1985, ApJ, 291, 152

Reynolds, S. P., Pavlov, G. G., Kargaltsev, O., et al. 2017, Space Sci. Rev., 207, 175

Roberts, D. A., Goss, W. M., Kalberla, P. M. W., Herbstmeier, U., \& Schwarz, U. J. 1993, A\&A, 274, 427

Roberts, M. S. E., Romani, R. W., Johnston, S., \& Green, A. J. 1999, ApJ, 515, 712

Roberts, M. S. E., Romani, R. W., \& Kawai, N. 2001, ApJS, 133, 451

Salter, C. J., Reynolds, S. P., Hogg, D. E., Payne, J. M., \& Rhodes, P. J. 1989, ApJ, 338, 171

Safi-Harb, S., \& Kumar, H. S. 2008, ApJ, 684, 532 
Safi-Harb, S., \& Kumar, H. S. 2012, Neutron Stars and Pulsars: Challenge and Opportunities after 80 yr, Proc. IAU Symp., 291

Seward, F. D., Harnden, F. R., Jr., Murdin, P., \& Clark, D. H. 1983, ApJ, 267, 698

Seward, F. D., Harnden, F. R., \& Helfand, D. J. 1984, ApJ, 287, L19

Sironi, L., \& Spitkovsky, A. 2011, ApJ, 741, 39

Sironi, L., \& Spitkovsky, A. 2012, Comput. Sci. Discovery, 5, 014014

Sironi, L., \& Spitkovsky, A. 2014, ApJ, 783, L21

Slane, P. O., Seward, F. D., Bandiera, R., Torii, K., \& Tsunemi, H. 1997, ApJ, 485,22

Slane, P., Zimmerman, E. R., Hughes, J. P., et al. 2004, ApJ, 601, 1045

Slane, P., Helfand, D. J., Reynolds, S. P., et al. 2008, ApJ, 676, L33

Spyrou, N. K., \& Stergioulas, N. 2002, A\&A, 395, 151

Tanaka, S. J., \& Takahara, F. 2010, ApJ, 715, 1248.

Tanaka, S. J., \& Takahara, F. 2011, ApJ, 741, 40

Tanaka, S. J., \& Takahara, F. 2013, MNRAS, 429, 2945

Taylor, J. H., \& Cordes, J. M. 1993, ApJ, 411, 674

Temim, T., Slane, P., Reynolds, S. P., Raymond, J. C., \& Borkowski, K. J. 2010, ApJ, 710, 309

Temim, T., Dwek, E., Arendt, R. G., et al. 2017, ApJ, 836, 129

Tian, W. W., \& Leathy, D. A. 2008, MNRAS, 391, 54

Torres, D. F., Cillis, A. N., \& Martín, J. 2013, ApJ, 763, L4
Torres, D. F., Cillis, A., Martín, J., \& de Oña Wilhelmi, E. 2014, J. High Energy Astrophys., 1, 31

Torii, K., Slane, P. O., Kinugasa, K., Hashimotodani, K., \& Tsunemi, H. 2000, PASJ, 52, 875

Tsujimoto, M., Guainazzi, M., Plucinsky, P. P., et al. 2011, A\&A, 525, A25

Ubertini, P., Bassani, L., Malizia, A., et al. 2005, ApJ, 629, L109

Van Etten, A., \& Romani, R. W. 2010, ApJ, 711, 1168

Velusamy, T., \& Becker, R. H. 1988, AJ, 95, 1162

Velusamy, T., Goss, W. M., \& Arnal, E. M. 1986, J. Astrophys. Astron., 7, 105

Wang, Q. D., \& Gotthelf E. V. 1998, A\&A, 494, 623

Wang, Z. R., Liu, J. Y., Gorenstein, P., \& Zombeck, M. V. 1986, Proc. Nineteenth IAU General Assembly, 7, Delhi, India, Highlights of Astronomy (Dordrecht: Reidel), 583

Weiler, K. W., \& Panagia, N. 1978, A\&A, 70, 419

Weltevrede, P., Johnston, S., \& Espinoza, C. M. 2011, MNRAS, 411, 1917

Williams, B. J., Borkowski, K. J., Reynolds, S. P., et al. 2008, ApJ, 687, 1054

Wilson, A. S., \& Weiler, K. W. 1976, A\&A, 53, 89

Winkler, P. F., Twelker, K., Reith, C. N., \& Long, K. S. 2009, ApJ, 692, 1489

Zhang, L., Chen, S. B., \& Fang, J. 2008, ApJ, 676, 1216

Zhang, L., Jiang, Z. J., \& Lin, G. F. 2009, ApJ, 699, 507

Zhu, B. T., Fang, J., \& Zhang, L. 2015, MNRAS, 451, 3145 


\section{Appendix A: Calculations of $R_{\mathrm{pwn}}(t)$ and $B_{\mathrm{pwn}}(t)$}

In this appendix, the calculations of $R_{\mathrm{pwn}}$ and $B_{\mathrm{pwn}}$ are briefly described in the frame of the model for dynamical and radiative evolution of a PWN inside an SNR presented in Gelfand et al. (2009).

\section{A.1. Calculation of $R_{p w n}(t)$}

To compute the radius of the PWN during the evolution process of the PWNe, the following initial conditions are required (Gelfand et al. 2009). The first is the equation of motion of the PWN for the pressure of the SNR ejecta $P_{\mathrm{snr}}\left(R_{\mathrm{pwn}}\right) \equiv 0$,

$M_{\mathrm{sw}, \mathrm{pwn}} \frac{\mathrm{d} v_{\mathrm{pwn}}}{\mathrm{d} t}=4 \pi R_{\mathrm{pwn}}^{2}\left[P_{\mathrm{pwn}}-\rho_{\mathrm{ej}}\left(R_{\mathrm{pwn}}\right) \times\left(v_{\mathrm{pwn}}-v_{\mathrm{ej}}\left(R_{\mathrm{pwn}}\right)\right)^{2}\right]$,

where $v_{\mathrm{pwn}}=\mathrm{d} R_{\mathrm{pwn}} / \mathrm{d} t, M_{\mathrm{sw}, \mathrm{pwn}}, P_{\mathrm{pwn}}$, and $R_{\mathrm{pwn}}$ are the velocity, mass, pressure, and radius of the PWN shell; $\rho_{\text {ej }}$ and $v_{\text {ej }}$ are the density and velocity of SNR ejecta and

$\frac{\mathrm{d} E_{\mathrm{pwn}}}{\mathrm{d} t}=\dot{E}-P_{\mathrm{pwn}} 4 \pi R_{\mathrm{pwn}}^{2} v_{\mathrm{pwn}}$

In the above treatment, the adiabatic losses dominate and the PWN is expanding with a constant velocity.

The second is expression of $R_{\text {pwn }}$ is

$R_{\mathrm{pwn}}(t)=1.44\left(\frac{E_{\mathrm{sn}}{ }^{3} \dot{E}_{0}{ }^{2}}{M_{\mathrm{ej}}{ }^{5}}\right)^{1 / 10} t^{6 / 5}$,

where $E_{\mathrm{sn}}$ is the explosion energy of the supernova, $\dot{E}_{0} \approx \dot{E}$, and $M_{\mathrm{ej}}$ is the mass of the SNR ejecta.

The PWN expands outwards due to the energy provided by the central pulsar and sweeps out the material of the SNR ejecta, so a thin shell is formed around the PWN. Because there is a pressure $P_{\mathrm{pwn}}$ inside the PWN and a pressure $P_{\mathrm{snr}}\left(R_{\mathrm{pwn}}\right)$ outside the PWN, the net force suffered by the PWN is $F_{\Delta \mathrm{P}}=$ $4 \pi R_{\mathrm{pwn}}{ }^{2}\left[P_{\mathrm{pwn}}-P_{\mathrm{snr}}\left(R_{\mathrm{pwn}}\right)\right]$, and the change rate of the momentum is

$\frac{\mathrm{d}}{\mathrm{d} t}\left(M_{\mathrm{sw}, \mathrm{pwn}} v_{\mathrm{pwn}}\right)=F_{\Delta \mathrm{P}}=4 \pi R_{\mathrm{pwn}}^{2}\left[P_{\mathrm{pwn}}-P_{\mathrm{snr}}\left(R_{\mathrm{pwn}}\right)\right]$.

In Eq. (A.4), the pressure of the PWN $P_{\text {pwn }}$ is calculated as follows. Assuming the PWN is mainly composed of a relativistic nonthermal lepton (electron/positron) plasma and magnetic field $E_{\mathrm{pwn}}=E_{\mathrm{pwn}, \mathrm{e}}+E_{\mathrm{pwn}, \mathrm{B}}$ (the spin-down power is described as $\left.L(t)=\dot{E}=\eta_{\mathrm{B}} \dot{E}+\eta_{\mathrm{e}} \dot{E}\right)$, where $E_{\mathrm{pwn}, \mathrm{e}}$ is the total energy contained in the leptons and is given by $E_{\mathrm{pwn}, \mathrm{e}}=\int_{0}^{E_{\max }} N(E, t) E \mathrm{~d} E$, and $E_{\mathrm{pwn}, \mathrm{B}}$ is the energy stored in the magnetic field of the PWN and reads $E_{\mathrm{pwn}, \mathrm{B}}=\left(B_{\mathrm{pwn}}^{2} / 8 \pi\right) V_{\mathrm{pwn}}$. The corresponding pressures contributed by the leptons and stored in the magnetic field of the $\mathrm{PWN}$ are $P_{\mathrm{pwn}, \mathrm{e}}=\left[\left(\gamma_{\mathrm{pwn}}-1\right) E_{\mathrm{pwn}, \mathrm{e}}\right] / V_{\mathrm{pwn}}=E_{\mathrm{pwn}, \mathrm{e}} /\left(4 \pi R_{\mathrm{pwn}}^{3}\right)$ and $P_{\mathrm{pwn}, \mathrm{B}}=B_{\mathrm{pwn}}^{2} / 8 \pi$, where $\gamma_{\mathrm{pwn}}=4 / 3$ and $V_{\mathrm{pwn}}=(4 / 3) R_{\mathrm{pwn}}^{3}$ is the volume of the PWN. Therefore, $P_{\mathrm{pwn}}=P_{\mathrm{pwn}, \mathrm{e}}+P_{\mathrm{pwn}, \mathrm{B}}$. $P_{\mathrm{snr}}\left(R_{\mathrm{pwn}}\right)$ is the pressure of the shocked material at $r=R_{\mathrm{pwn}}$ and its calculation (see Appendix A in Gelfand et al. 2009).

If the density, velocity, pressure of the ejecta, and the inner pressure of the PWN at some time $t$ are given, then the radius and velocity of the PWN shell are calculated using above expressions. The calculation process here is the same as that in Gelfand et al. (2009, see their Sect. 2.2, in details).

\section{A.2. Calculation of $B_{\text {pwn }}(t)$}

From the relation of $B_{\mathrm{pwn}}$ and $L(t)$ is given by

$\frac{\mathrm{d} E_{\mathrm{pwn}, \mathrm{B}}(t)}{\mathrm{d} t}=\eta_{\mathrm{B}} L(t)-E_{\mathrm{pwn}, \mathrm{B}}(t) \frac{1}{R_{\mathrm{pwn}}(t)} \frac{\mathrm{d} R_{\mathrm{pwn}}(t)}{\mathrm{d} t}$,

the following equation can be derived:

$\frac{\mathrm{d}\left[E_{\mathrm{pwn}, \mathrm{B}}(t) R_{\mathrm{pwn}}(t)\right]}{\mathrm{d} t}=\eta_{\mathrm{B}} R_{\mathrm{pwn}}(t) L(t)$.

Integrating Eq. (A.5) from $t=0$ to $t$ and noting that $E_{\mathrm{pwn}, \mathrm{B}}(t)=0$ at $t=0$, the magnetic field $B_{\text {pwn }}(t)$ is given by

$B_{\mathrm{pwn}}(t)=\left(\frac{1}{R_{\mathrm{pwn}}(t)}\right)^{2} \sqrt{6 \eta_{\mathrm{B}} \int_{0}^{t} L\left(t^{\prime}\right) R_{\mathrm{pwn}}\left(t^{\prime}\right) \mathrm{d} t^{\prime}}$.

\section{Appendix B: Basic properties of PWNe in the sample}

\section{B.1. Group 1: young PWNe}

$N$ 158A. It is also known as G279.7-31.5, and is powered by an energetic pulsar PSR B0540-69 with a rotation period of $50.5 \mathrm{~ms}$, a period derivative of $4.79 \times 10^{-13} \mathrm{~s} \mathrm{~s}^{-1}$ (Seward et al. 1984; Livingstone et al. 2005b; Ferdman et al. 2015), and a braking index $n$ for the PSR B0540-69 is 2.08 (Kaaret et al. 2001), very recent measurement shows $n=2.129 \pm 0.012$ (Ferdman et al. 2015), which is used here. Therefore, the pulsar has a characteristic age of $\sim 1672 \mathrm{yr}$, and a spin-down luminosity $L(t)=1.47 \times 10^{38} \mathrm{erg} \mathrm{s}^{-1}$. The system lies at a distance of $49 \mathrm{kpc}$ (Seward et al. 1984; Taylor \& Cordes 1993). Meanwhile, according to Manchester et al. (1993), the physical size of the N 158A is 0.7 pc. Observationally, N 158A has been detected in radio (Manchester et al. 1993), IR and optical (Mignani et al. 2012), and X-ray bands (Kaaret et al. 2001; Campana et al. 2008), but it was not detected in $\mathrm{GeV}$ and $\mathrm{TeV}$ $\gamma$-ray bands. The age of the system is $\sim 760 \mathrm{yr}$ by measurements of the expansion velocity of the SNR shell in the optical spectral band (Reynolds 1985; Kirshner et al. 1989; Chevalier 2005), and the mass of $20-25 M_{\odot}$ for the progenitor star is inferred by Williams et al. (2008). The parameters used in our calculation are $E_{\mathrm{sn}}=2.0 \times 10^{51} \mathrm{erg}, M_{\mathrm{ej}}=23.0 M_{\odot}$, and $n_{\mathrm{H}}=0.01 \mathrm{~cm}^{-3}$. In Martin et al. (2014), $U_{\mathrm{IR}}=5.0 \mathrm{eV} \mathrm{cm}^{-3}$ and $U_{\mathrm{OPT}}=0.2 \mathrm{eV} \mathrm{cm}^{-3}$ were used to compute the energy density needed for the PWN to be detected by CTA. Here, $U_{\mathrm{IR}}=1.0 \mathrm{eV} \mathrm{cm}^{-3}$ and $U_{\mathrm{OPT}}=1.0 \mathrm{eV} \mathrm{cm}^{-3}$ are used in our calculation.

PWN G21.5-0.9 or HESS J1833-105. It is powered by an energetic PSR J1833-1034. The pulsar has a $61.8 \mathrm{~ms}$ rotation period, a period derivative of $2.02 \times 10^{-13} \mathrm{~s} \mathrm{~s}^{-1}$ (Gupta et al. 2005; Camilo et al. 2006), and $n=3.0$ is assumed. Thus, the pulsar has a characteristic age $\tau_{\mathrm{c}}=4850 \mathrm{yr}$ and spin-down power $L(t)=3.4 \times 10^{37} \mathrm{erg} \mathrm{s}^{-1}$. According to Camilo et al. (2006), the distance of the system is $4.7 \pm 0.4 \mathrm{kpc}$; the same value was obtained by Tian \& Leathy (2008). Here the distance of $4.1 \mathrm{kpc}$ (Abdalla et al. 2017) is used. Observationally, PWN G21.5-0.9 is detected in radio (Salter et al. 1989; Morsi \& Reich 1987; Wilson \& Weiler 1976; Becker \& Kundu 1976), IR (Gallant \& Tuffs 1998, 1999), X-ray (De Rosa et al. 2009; Tsujimoto et al. 2011; Nynka et al. 2014), and TeV $\gamma$ ray bands by HESS (Djannati-Atai et al. 2008). Although Wang et al. (1986) suggested that the age of PWN G21.5-0.9 is about 2000 yr because it might be the historical supernova of 
$48 \mathrm{BC}$, Bietenholz \& Bartel (2008) estimated $T_{\text {age }} \sim 900 \mathrm{yr}$ through the observation of the expansion rate of the PWN. Following Bietenholz \& Bartel (2008) or Tanaka \& Takahara (2011), $T_{\text {age }}=900 \mathrm{yr}$ is assumed here. The parameters used in our calculation are $n_{\mathrm{H}}=0.1 \mathrm{~cm}^{-3}$, which is constrained to be between 0.1 and $0.4 \mathrm{~cm}^{-3}$ by Matheson \& Safi-Harb (2005), and $M_{\mathrm{ej}}=8.0 M_{\odot}$. The values $U_{\mathrm{IR}}=1.4 \mathrm{eV} \mathrm{cm}^{-3}$ and $U_{\mathrm{OPT}}=$ $5.0 \mathrm{eV} \mathrm{cm}^{-3}$ given in the GALPROP code of Porter et al. (2006) are used.

Crab nebula. It is a famous PWN that has been widely studied. The details of the observed and derived properties for the Crab nebula can be found in many papers (e.g., Torres et al. 2014; Zhu et al. 2015).

Kes 75. It is also known as HESS J1846-029 or G29.70.3 , which is a typical composite supernova remnant. The central pulsar is PSR 1846-0258 (Gotthelf et al. 2000), and it has a rotational period $P=324 \mathrm{~ms}$, a period derivative $\dot{P}=$ $7.08 \times 10^{-12} \mathrm{~s} \mathrm{~s}^{-1}$, and a braking index $n=2.65 \pm 0.01$ (Livingstone et al. 2006). Very recently, the braking index $n=$ $2.19 \pm 0.03$ was measured by Archibald et al. (2015); this value is applied in our paper. Thus, the pulsar has a characteristic age $\tau_{\mathrm{c}}=730 \mathrm{yr}$ and its spin-down power $L(t)=8.21 \times 10^{36} \mathrm{erg} \mathrm{s}^{-1}$. Leahy \& Tian (2008) estimated a distance for the system between 5.1 to $7.5 \mathrm{kpc}$; the distance of $5.8 \mathrm{kpc}$ (Abdalla et al. 2017 ) is adopted in our model. Observationally, Kes 75 has been detected in radio band (Salter et al. 1989; Bock \& Gaensler 2005), X-ray band (Helfand et al. 2003), and TeV $\gamma$-ray band by HESS (Djannati-Atai et al. 2008). The ambient medium density of $1 \mathrm{~cm}^{-3}$ is estimated by Safi-Harb \& Kumar (2012). Recently, the ambient medium density is constrained to be between 0.005 and $0.1 \mathrm{~cm}^{-3}$ by Gelfand et al. (2014); here, $0.1 \mathrm{~cm}^{-3}$ is used. The PSR 1846-0258 is very young; the actual age was constrained to be between 980 and $1770 \mathrm{yr}$ by Mereghetti et al. (2002). Here, the age of $1000 \mathrm{yr}$ and $M_{\mathrm{ej}}=10.0 M_{\odot}$ are used. The values $U_{\mathrm{IR}}=1.2 \mathrm{eV} \mathrm{cm}^{-3}$ and $U_{\mathrm{OPT}}=2.0 \mathrm{eV} \mathrm{cm}^{-3}$ are taken from Tanaka \& Takahara (2011).

G310.6-1.6. It is powered by an energetic pulsar PSR $\mathrm{J} 1400-6325$, which has a rotation period of $31.18 \mathrm{~ms}$, a period derivative $3.89 \times 10^{-14} \mathrm{~s} \mathrm{~s}^{-1}$ (Renaud et al. 2010), and $n=3.0$ is assumed. Thus, these parameters derive the characteristic age $\tau_{\mathrm{c}}=12700 \mathrm{yr}$ and spin-down power $L(t)=$ $5.1 \times 10^{37} \mathrm{erg} \mathrm{s}^{-1}$. The system lies at a distance of $7 \mathrm{kpc}$ (Renaud et al. 2010; Tanaka \& Takahara 2013). Observationally, G310.6-1.6 has been measured in radio band (Murphy et al. 2007; Griffith \& Wright 1993; Condon et al. 1993; Duncan et al. 1995), X-ray band (Renaud et al. 2010), and TeV $\gamma$-ray band by HESS (e.g., Khélifi et al. 2008). The parameters used here are $M_{\mathrm{ej}}=13.0 M_{\odot}$, and $n_{\mathrm{H}}=0.01 \mathrm{~cm}^{-3}$. The values $U_{\mathrm{IR}}=0.3 \mathrm{eV} \mathrm{cm}^{-3}$ and $U_{\mathrm{OPT}}=0.3 \mathrm{eV} \mathrm{cm}^{-3}$ are taken from Tanaka \& Takahara (2013).

$3 C$ 58. It is also known as SNR G130.7+3.1 or SN 1181 , which is a composite SNR (Weiler \& Panagia 1978). Its central pulsar is PSR J0205+6449, which is detected in radio, X-ray, and $\gamma$-ray bands. The pulsar has a rotation period $65.7 \mathrm{~ms}$, a period derivative of $1.93 \times 10^{-13} \mathrm{~s} \mathrm{~s}^{-1}$ (Murray et al. 2002; Camilo et al. 2002b; Livingstone et al. 2009), and $n=3.0$ is assumed. These parameters derive a characteristic age of $\sim 5397 \mathrm{yr}$ and a spin-down luminosity $L(t)=2.68 \times 10^{37} \mathrm{erg} \mathrm{s}^{-1}$. According to Roberts et al. (1993), 3C 58 is located at a distance of $3.2 \mathrm{kpc}$ (Roberts et al. 1993) or $2 \mathrm{kpc}$ (Kothes et al. 2010; Kothes 2013). Here, the distance of $2 \mathrm{kpc}$ is adopted. Observationally, 3C 58 has been detected in radio (Green 1986; Morsi \& Reich 1987; Salter et al. 1989), IR (Green 1994; Slane et al. 2008),
X-ray (Torii et al. 2000), and GeV by Fermi-LAT (Abdo et al. 2013; Ackermann et al. 2013) and TeV bands by MAGIC (Aleksić et al. 2014). Chevalier (2005) estimated that the age of the system is $\sim 2400 \pm 500$ yr by the PWN evolution and energetics, so $2400 \mathrm{yr}$ is used in our paper. The parameters used in our calculation are $M_{\mathrm{ej}}=8.0 M_{\odot}$, and $n_{\mathrm{H}}=0.01 \mathrm{~cm}^{-3}$. The values $U_{\mathrm{IR}}=0.3 \mathrm{eV} \mathrm{cm}^{-3}$ and $U_{\mathrm{OPT}}=0.3 \mathrm{eV} \mathrm{cm}^{-3}$ are taken from Tanaka \& Takahara (2013).

\section{B.2. Group 2: evolved PWNe}

HESS J1813-178 or G12.8-0.0. It is powered by an energetic pulsar PSR J1813-1749, which is the third most energetic pulsar in the Galaxy (Halpern et al. 2012). The pulsar has a rotation period of $44.7 \mathrm{~ms}$ (Gotthelf \& Halpern 2009), a period derivative of $1.265 \times 10^{-13} \mathrm{~s} \mathrm{~s}^{-1}$ (Halpern et al. 2012), and $n=3.0$ is assumed. These parameters lead to a characteristic age of $\sim 5600 \mathrm{yr}$ and a spin-down luminosity $L(t)=5.6 \times 10^{37} \mathrm{erg} \mathrm{s}^{-1}$. According to Brogan et al. (2005) and Halpern et al. (2012), the distance of the PWN is $4.7 \mathrm{kpc}$, and the corresponding radius of $4.0 \pm 0.3$ pc (Funk et al. 2007; Abdalla et al. 2017). Observationally, Brogan et al. (2005) presented the discovery of nonthermal radio emission of the radio shell (SNR G12.8-0.0) that is spatially coincident with HESS J1813-178, so we assume that the radio emission comes from HESS J1813-178, which was observed in X-ray band (Funk et al. 2007; Ubertini et al. 2005), $\mathrm{GeV}$ band by Fermi-LAT (Acero et al. 2013), and TeV $\gamma$-ray band by HESS (Aharonian et al. 2006b). In our model, we assume an age of $2500 \mathrm{yr}$. The parameters used in our calculation are the density of ambient medium of $1.0 \mathrm{~cm}^{-3}$ (Brogan et al. $2005)$, and $M_{\mathrm{ej}}=8.0 M_{\odot}$. For the soft seed photons, $U_{\mathrm{IR}}=$ $1.0 \mathrm{eV} \mathrm{cm}^{-3}$ and $U_{\mathrm{OPT}}=1.5 \mathrm{eV} \mathrm{cm}^{-3}$ are used in Fang \& Zhang (2010b). Here, $U_{\mathrm{IR}}=0.1 \mathrm{eV} \mathrm{cm}^{-3}$ and $U_{\mathrm{OPT}}=0.5 \mathrm{eV} \mathrm{cm}^{-3}$ are used to reproduce observed $\gamma$-ray SED.

G54.1+0.3. It is also known as VER J1930+188, which was first discovered by Reich et al. (1985). The PWN is powered by an energetic pulsar PSR J1930+1852, which was observed in radio (Camilo et al. 2002a) and X-ray bands (Lu et al. 2007). The pulsar has a period of $136 \mathrm{~ms}$, a period time derivative $7.51 \times$ $10^{-13} \mathrm{~s} \mathrm{~s}^{-1}$ (Camilo et al. 2002a), and $n=3.0$ is assumed. These parameters lead to a characteristic age of $\sim 2872 \mathrm{yr}$, and the spindown power $L(t)=1.16 \times 10^{37} \mathrm{erg} \mathrm{s}^{-1}$. PWN G54.1+0.3 has a distance of $6.2_{-0.6}^{+1.0} \mathrm{kpc}$ (Leahy et al. 2008). Here, the distance of $7.0 \mathrm{kpc}$ (Abdalla et al. 2017) is used. Observationally, the PWN has been observed in radio band (Green 1985; Velusamy et al. 1986; Velusamy \& Becker 1988; Lang et al. 2010), X-ray band (Lu et al. 2001, 2002; Bocchino et al. 2010), and TeV band by HESS (Acciari et al. 2010). The age of G54.1+0.3 is estimated to be between 1500 and 6000 yr (Camilo et al. 2002a). Recently, the age is constrained to be between 2100 and $3600 \mathrm{yr}$ (Chevalier 2005; Bocchino et al. 2010; Gelfand et al. 2015). Here $T_{\text {age }}=$ $2600 \mathrm{yr}$ is used. The ejected mass $M_{\mathrm{ej}}=20 M_{\odot}$, which is constrained between 16 and $27 M_{\odot}$ (Temim et al. 2010, 2017) and $n_{\mathrm{H}}=1.0 \mathrm{~cm}^{-3}$ are used here. The values $U_{\mathrm{IR}}=0.8 \mathrm{eV} \mathrm{cm}^{-3}$ and $U_{\mathrm{OPT}}=1.1 \mathrm{eV} \mathrm{cm}^{-3}$ are taken from the GALPROP code of Porter et al. (2006).

G292.0+1.8. It is also known as MSH 11-54 or HI 122-59. The system is powered by an energetic pulsar PSR J11245916 with a rotation period of $135.31 \mathrm{~ms}$ and a period derivative of $7.47 \times 10^{-13} \mathrm{~s} \mathrm{~s}^{-1}$ (Camilo et al. 2002a); $n=3.0$ is assumed. Thus, the pulsar has a characteristic age of $\sim 2872 \mathrm{yr}$ and a spin-down luminosity $L(t)=1.19 \times 10^{37} \mathrm{erg} \mathrm{s}^{-1}$. The system lies at a distance of $6.0 \mathrm{kpc}$ (Winkler et al. 2009). The 
radius of the PWN is about 3 pc (Bhalerao et al. 2015). Observationally, G292.0+1.8 has been observed in radio band (Gaensler \& Wallace 2003), X-ray band (Hughes et al. 2001), and $\mathrm{GeV}$ band with an upper limit (Ackermann et al. 2011). The age of the system is uncertain, and ranges from $\leq 1600 \mathrm{yr}$ (Murdin \& Clark 1979) to 23000 yr (Winkler et al. 2009); here $T_{\text {age }}=2700 \mathrm{yr}$ is used, which is consistent with Tanaka \& Takahara (2013). The values of $n_{\mathrm{H}}=0.5 \mathrm{~cm}^{-3}$ and $M_{\mathrm{ej}}=18.0 M_{\odot}$ are adopted. For the soft seed photons, $U_{\mathrm{IR}}=0.3 \mathrm{eV} \mathrm{cm}^{-3}, U_{\mathrm{OPT}}=0.3 \mathrm{eV}$ and $U_{\mathrm{IR}}=0.42 \mathrm{eV} \mathrm{cm}^{-3}$, $U_{\mathrm{OPT}}=0.7 \mathrm{eV}$ are applied in Tanaka \& Takahara (2013) and Martin et al. (2014), respectively. Here, $U_{\mathrm{IR}}=1.0 \mathrm{eV} \mathrm{cm}^{-3}$ and $U_{\mathrm{OPT}}=0.7 \mathrm{eV} \mathrm{cm}^{-3}$ are used.

G0.9+0.1 or HESS J1747-281. It is powered by an energetic pulsar PSR J1747-2809 with a rotation period of $52.2 \mathrm{~ms}$ and a period derivative of $1.56 \times 10^{-13} \mathrm{~s} \mathrm{~s}^{-1}$ (Camilo et al. 2009), and $n=3.0$ is assumed. This pulsar has a characteristic age $\tau_{\mathrm{c}}=5305 \mathrm{yr}$ and the spin-down power $L(t)=$ $4.32 \times 10^{37} \mathrm{erg} \mathrm{s}^{-1}$. The distance of the system is likely in the range from $8.0 \mathrm{kpc}$ to $16 \mathrm{kpc}$, due to the uncertainty of the electron density model toward the distant inner Galactic regions (Dubner et al. 2008). Here, the distance of $13.3 \mathrm{kpc}$ (Abdalla et al. 2017) is used. The PWN was detected in radio (Dubner et al. 2008), X-ray (Porquet et al. 2003), and TeV $\gamma$-ray bands by HESS (Aharonian et al. 2005b). Camilo et al. (2009) estimated the age of the system in the range between $2000 \mathrm{yr}$ and $3000 \mathrm{yr}$. Here the age of the PWN is assumed to be $3000 \mathrm{yr}$; the density of the ambient medium of $0.01 \mathrm{~cm}^{-3}$ and $M_{\mathrm{ej}}=14.0 M_{\odot}$ are used in our calculation. The values $U_{\mathrm{IR}}=3.8 \mathrm{eV} \mathrm{cm}^{-3}$ and $U_{\mathrm{OPT}}=25.0 \mathrm{eV} \mathrm{cm}^{-3}$ given in Torres et al. (2014) are used.

MSH 15-52. A typical composite supernova remnant, which is also known as HESS J1514-591 or SNR G320.4-1.2 (Caswell et al. 1981). The system was first discovered as an extended nonthermal radio source by Mills et al. (1961). The PWN is powered by an energetic pulsar PSR B1509-58 (Gaensler et al. 2008), and it has a $150 \mathrm{~ms}$ rotation period, a period derivative of $1.5 \times 10^{-12} \mathrm{~s} \mathrm{~s}^{-1}$, and a braking index $n=2.839 \pm 0.003$ (Livingstone et al. 2005a). Therefore, the pulsar has a characteristic age $\tau_{\mathrm{c}} \sim 1585 \mathrm{yr}$ and spin-down power $L(t)=1.75 \times$ $10^{37} \mathrm{erg} \mathrm{s}^{-1}$. This system is located at a distance of $5.2 \pm 1.4 \mathrm{kpc}$ based on the HI absorption measurement (Gaensler et al. 1999). It is consistent with the vale of $4.2 \pm 0.6 \mathrm{kpc}$ derived from the dispersion measure Cordes \& Lazio (2002). Here, the distance of $4.4 \mathrm{kpc}$ (Abdalla et al. 2017) is used. Observationally, MSH 15-52 has been detected in radio band (Gaensler et al. 1999, 2008), X-ray band (Mineo et al. 2001; Forot et al. 2006), $\mathrm{GeV}$ band by Fermi-LAT (Abdo et al. 2010), and TeV $\gamma$-ray band by HESS (Aharonian et al. 2005a). Although the spindown age is $\sim 1600 \mathrm{yr}$, the true age of the system could be much older than the spin-down age (Seward et al. 1983; Gvaramadze 2001). To reconcile the age of the system, the braking index has to be $<2.0$. Here $T_{\text {age }}=4000 \mathrm{yr}$ and $n=1.4$ are assumed. The density of ambient medium $n_{\mathrm{H}}=0.01 \mathrm{~cm}^{-3}$ and the ejected mass $M_{\mathrm{ej}}=6.0 M_{\odot}$ are adopted; $U_{\mathrm{IR}}=1.2 \mathrm{eV} \mathrm{cm}^{-3}$ and $U_{\mathrm{OPT}}=2.2 \mathrm{eV} \mathrm{cm}^{-3}$ are taken from the GALPROP code of Porter et al. (2006).

$N 157 B$. It was the first extragalactic PWN to be detected in TeV $\gamma$-rays with HESS (Abramowski et al. 2012a, 2015). Its central pulsar is PSR J0537-6910 (Marshall et al. 1998). The pulsar has a rotation period of $16.12 \mathrm{~ms}$ and a period derivative of $5.13 \times 10^{-14} \mathrm{~s} \mathrm{~s}^{-1}$ (Spyrou \& Stergioulas 2002; Manchester et al. 2005b); $n=3.0$ is assumed. Thus, the pulsar has a characteristic age of $\sim 4982 \mathrm{yr}$, and a spin-down luminosity
$L(t)=4.82 \times 10^{38} \mathrm{erg} \mathrm{s}^{-1}$. The system has an estimated distance of $48 \mathrm{kpc}$ (Macri et al. 2006), and the radius of the PWN is $10.6 \mathrm{pc}$ (Lazendic et al. 2000). However, this radius is not very well defined, and it could include parts of the remnant. Following Abdalla et al. (2017), the distance of $53.7 \mathrm{kpc}$ is used here. Observationally, this PWN has been detected in radio band (Lazendic et al. 2000), X-ray band (Chen et al. 2006), and $\mathrm{TeV} \gamma$-ray band by HESS (Abramowski et al. 2012a, 2015). For the whole system, the Sedov age of $\sim 5000 \mathrm{yr}$ was estimated by Wang \& Gotthelf (1998). Here, $T_{\text {age }}=4600 \mathrm{yr}$, which is consistent with Martin et al. (2014), and $n_{\mathrm{H}}=0.03 \mathrm{~cm}^{-3}$ and $M_{\mathrm{ej}}=20.0 M_{\odot}$, which is consistent with Chen et al. (2006), are used. For far-IR photons, according to the OB association LH99 and the nearby star-forming region 30 Doradus, using observations from Spitzer (Indebetouw et al. 2009), the infrared photon fields are modeled as blackbody radiation with a temperature of $80 \mathrm{~K}$ and an energy density of $8.9 \mathrm{eV} \mathrm{cm}^{-3}$ for LH99, and a temperature of $88 \mathrm{~K}$ and an energy density of $2.7 \mathrm{eV} \mathrm{cm}^{-3}$ for 30 Doradus. These are only upper limits to the infrared fields since the distances of N157B to these objects are unknown. Here, $U_{\mathrm{IR}}=5.0 \mathrm{eV} \mathrm{cm}^{-3}$ and $U_{\mathrm{OPT}}=4.0 \mathrm{eV} \mathrm{cm}^{-3}$ are used.

\section{B.3. Group 3: mature/old PWNe}

HESS J1356-645 or G309.9-2.51. It is powered by an energetic pulsar PSR J1357-6429 with a $166 \mathrm{~ms}$ rotation period, a $3.6 \times 10^{-13} \mathrm{~s} \mathrm{~s}^{-1}$ period derivative (Camilo et al. 2004; Lorimer et al. 2006), and $n=3.0$ is assumed. So the characteristic age of this pulsar is $\sim 7310 \mathrm{yr}$ and the spin-down luminosity is $L(t)=3.1 \times 10^{36} \mathrm{erg} \mathrm{s}^{-1}$. Its distance is estimated to be $2.5 \mathrm{kpc}$ (Cordes \& Lazio 2002; Chang et al. 2012) and its radius is $10.1 \pm$ 0.9 pc (Abdalla et al. 2017). Observationally, the PWN has been observed at radio band (Duncan et al. 1995; Griffith \& Wright 1993; Murphy et al. 2007), X-ray band (Abramowski et al. 2011), GeV $\gamma$-ray band by Fermi-LAT (Acero et al. 2013), and TeV $\gamma$-ray band by HESS (Abramowski et al. 2011). Because the age of the system is not clear, $T_{\text {age }}=6500 \mathrm{yr}$ is used here, which is similar to Torres et al. (2014). The values $M_{\mathrm{ej}}=7.0 M_{\odot}$ and $n_{\mathrm{H}}=0.05 \mathrm{~cm}^{-3}$ are assumed; $U_{\mathrm{IR}}=0.4 \mathrm{eV} \mathrm{cm}^{-3}$ and $U_{\mathrm{OPT}}=0.5 \mathrm{eV} \mathrm{cm}^{-3}$ are the same as those in Torres et al. (2014).

CTA 1. It is also known as VER J0006+727 or G $119.5+$ 10.2, which was first discovered by Harris \& Roberts (1960). The PWN is powered by PSR J0007+7303 with a rotation period of $316.86 \mathrm{~ms}$, a period derivative of $3.614 \times 10^{-13} \mathrm{~s} \mathrm{~s}^{-1}$ (Abdo et al. 2008), and $n=3.0$ is assumed. Thus, the pulsar has a characteristic age of $\sim 13900 \mathrm{yr}$ and a spin-down luminosity $L(t)=4.48 \times 10^{35} \mathrm{erg} \mathrm{s}^{-1}$. CTA 1 has a distance of $1.4 \pm 0.3 \mathrm{kpc}$ (Pineault et al. 1993). The radius of the CTA 1 is estimated to be $R_{\text {pwn }} \sim 7.2$ pc because of the large synchrotron nebula observed by Slane et al. (1997, 2004), Halpern et al. (2004). Here, $R_{\text {pwn }}=6.6 \pm 0.5$ (Abdalla et al. 2017) is used. Observationally, CTA 1 has been detected in radio band (Aliu et al. 2013), X-ray band (Slane et al. 1997, 2004), GeV $\gamma$-ray band by FermiLAT (Abdo et al. 2012), and TeV $\gamma$-ray band by VERITAS (Aliu et al. 2013). The parameters used here are $T_{\text {age }}=7500 \mathrm{yr}$ (which is between the values of $5000 \mathrm{yr}$ and $15000 \mathrm{yr}$ found by Pineault et al. 1993; Slane et al. 2004), $M_{\mathrm{ej}}=8.0 M_{\odot}$, and $n_{\mathrm{H}}=0.1 \mathrm{~cm}^{-3}$. The values $U_{\mathrm{IR}}=0.3 \mathrm{eV} \mathrm{cm}^{-3}$ and $U_{\mathrm{OPT}}=$ $0.6 \mathrm{eV} \mathrm{cm}^{-3}$ are taken from the GALPROP code of Porter et al. (2006).

HESS J1418-609. It is also known as Rabbit or G313.3+0.1. The PWN is powered by PSR J1418-6058 (Acero et al. 2013). The pulsar has a rotation period of $110.6 \mathrm{~ms}$, a period derivative of $1.69 \times 10^{-13} \mathrm{~s} \mathrm{~s}^{-1}$ (Abdo et al. 2013), and $n=3.0$ is 
assumed. This pulsar has a characteristic age of $\sim 10380 \mathrm{yr}$ and a spin-down luminosity $L(t)=4.93 \times 10^{36} \mathrm{erg} \mathrm{s}^{-1}$. The distance of the system is $5.0 \mathrm{kpc}(\mathrm{Ng}$ et al. 2005; Aharonian et al. 2006a), which is used here. The radius of the system was estimated as $6.5 \pm 0.3 \mathrm{pc}$ (Kishishita et al. 2012) or $9.4 \pm 0.9$ (Abdalla et al. 2017). HESS J1418-609 has been detected in radio band (Roberts et al. 1999), X-ray band (Roberts et al. 2001; Ng et al. 2005; Kishishita et al. 2012), GeV $\gamma$-ray band by Fermi-LAT (Acero et al. 2013), and TeV $\gamma$-ray band by HESS (Aharonian et al. 2006a). So far, the age of HESS J1418-609 has not been clearly determined. The parameters used in our calculation are $T_{\text {age }}=8000 \mathrm{yr}, M_{\mathrm{ej}}=10.0 M_{\odot}$, and $n_{\mathrm{H}}=0.2 \mathrm{~cm}^{-3}$; $U_{\mathrm{IR}}=0.4 \mathrm{eV} \mathrm{cm}^{-3}$ and $U_{\mathrm{OPT}}=1.0 \mathrm{eV} \mathrm{cm}^{-3}$ are used to reproduce observed $\gamma$-ray SED here.

HESS J1420-607. It is also known as K3 PWN. The central pulsar is PSR J1420-6048 with a rotation period of $68 \mathrm{~ms}$, a period derivative of $8.3 \times 10^{-14} \mathrm{~s} \mathrm{~s}^{-1}$ (D'Amico et al. 2001; Roberts et al. 2001; Ng et al. 2005; Kishishita et al. 2012), and $n=3.0$ is assumed. Therefore, the characteristic age of $\sim 13000 \mathrm{yr}$ and the spin-down luminosity $L(t)=1.0 \times$ $10^{37} \mathrm{erg} \mathrm{s}^{-1}$ are derived. The distance of the system is estimated as $d=5.6 \mathrm{kpc}$ (Cordes \& Lazio 2002), and the PWN radius is $7.9 \pm 0.6 \mathrm{pc}$ (Abdalla et al. 2017), which is consistent with Kishishita et al. (2012). HESS J1420-607 has been detected in radio band (Van Etten \& Romani 2010), X-ray band (Van Etten \& Romani 2010; Kishishita et al. 2012), GeV $\gamma$-ray band by Fermi-LAT Acero et al. (2013), and TeV $\gamma$-ray band by HESS Aharonian et al. (2006a). The age of the system is not clear. The parameters used here are $T_{\mathrm{age}}=8500 \mathrm{yr}, n_{\mathrm{H}}=$ $0.5 \mathrm{~cm}^{-3}$, and $M_{\mathrm{ej}}=15.0 M_{\odot} ; U_{\mathrm{IR}}=0.3 \mathrm{eV} \mathrm{cm}^{-3}$ and $U_{\mathrm{OPT}}=$ $0.3 \mathrm{eV} \mathrm{cm}^{-3}$ are used to reproduce observed $\gamma$-ray SED here.

HESS J1119-614 or G292.2-0.5. The PWN associates with the pulsar J1119-6127 (Caswell et al. 2004), which was discovered by Camilo et al. (2000). The pulsar has a rotational period of $408 \mathrm{~ms}$, a period derivative of $4 \times 10^{-12} \mathrm{~s} \mathrm{~s}^{-1}$, and the braking index $n=2.684 \pm 0.002$ (Weltevrede et al. 2011), which derives the characteristic age $\tau_{\mathrm{c}}=1617 \mathrm{yr}$ and spin-down power $L(t)=2.32 \times 10^{36} \mathrm{erg} \mathrm{s}^{-1}$. It lies at a distance of $8.4 \pm 0.4 \mathrm{kpc}$ (Caswell et al. 2004). The radius of the system is $14 \pm 2 \mathrm{pc}$ (Abdalla et al. 2017). Observationally, this PWN is detected in
X-ray band (Gonzalez \& Safi-Harb 2003; Safi-Harb \& Kumar 2008) and the X-ray unabsorbed flux between 0.5 and $7 \mathrm{keV}$ is $2.5 \times 10^{-14} \mathrm{erg} \mathrm{cm}^{-2} \mathrm{~s}^{-1}$ and spectral index $1.4 \pm_{0.9}^{0.8}$. It is detected in GeV band by Fermi-LAT (Acero et al. 2013), and TeV band by HESS (e.g., Abdalla et al. 2017). Here the whole SNR G292.2-0.5 flux measurement at $1.4 \mathrm{GHz}$ flux density of $5.6 \pm 0.3 \mathrm{Jy}$ and 2.5 flux density of $1.6 \pm 0.1 \mathrm{Jy}$ (Crawford et al. 2001) are taken as a safe upper limit for the PWN radio emission. The age of HESS J1119-614 lies between $4200 \mathrm{yr}$ (free expansion phase) and $7100 \mathrm{yr}$ (Sedov phase; Kumar et al. 2012). We note that $T_{\text {age }}=4200 \mathrm{yr}$ and $n=1.7$ are used in Torres et al. (2014). These ages are older than the characteristic age $\sim 1617 \mathrm{yr}$. Here $T_{\text {age }}=9000 \mathrm{yr}$ and $n=1.2$ are assumed. The parameters used in our calculation are $M_{\mathrm{ej}}=35.0 M_{\odot}$ and $n_{\mathrm{H}}=0.06 \mathrm{~cm}^{-3}$; $U_{\mathrm{IR}}=0.3 \mathrm{eV} \mathrm{cm}^{-3}$ and $U_{\mathrm{OPT}}=0.7 \mathrm{eV} \mathrm{cm}^{-3}$ are taken from the GALPROP code of Porter et al. (2006).

HESS J1303-631. It was first discovered in the VHE band by Aharonian et al. (2005c) and is associated with the pulsar J13016305 (Abramowski et al. 2012b). The pulsar has a $184 \mathrm{~ms}$ rotation period, a $2.65 \times 10^{-13} \mathrm{~s} \mathrm{~s}^{-1}$ period derivative, and $n=$ 3.0 is assumed. So a characteristic age of $\sim 11000 \mathrm{yr}$ and a spin-down luminosity $L(t)=1.7 \times 10^{36} \mathrm{erg} \mathrm{s}^{-1}$ are derived (Manchester et al. 2005a; Abramowski et al. 2012b). The pulsar's distance was estimated to be $15.8 \mathrm{kpc}$ by Taylor \& Cordes (1993) and $6.6 \mathrm{kpc}$ by Cordes \& Lazio (2002). The distance of $6.6 \mathrm{kpc}$ is used here. The radius of the system was estimated as 20 pc by (Abramowski et al. 2012b) and 20.6 \pm 1.7 (Abdalla et al. 2017). Observationally, HESS J1303-631 has been detected in radio band (Condon et al. 1993), X-ray band (Abramowski et al. 2012b), GeV $\gamma$-ray band by Fermi-LAT (Acero et al. 2013), and TeV $\gamma$-ray band by HESS (Abramowski et al. 2012b); the X-ray unabsorbed flux between 2 and $10 \mathrm{KeV}$ is $1.6 \times 10^{-13}$ and the spectral index is unknown. Here, the value is assumed as a safe upper limit. The multiband spectrum of HESS J1303631 was first studied by a simple stationary leptonic model (Abramowski et al. 2012b). The age of the system is not clear; here $T_{\text {age }}=13000 \mathrm{yr}$ and $n=2.5$ are assumed. In our calculations, $M_{\mathrm{ej}}=9.0 M_{\odot}$ and $n_{\mathrm{H}}=0.02 \mathrm{~cm}^{-3} ; U_{\mathrm{IR}}=6.0 \mathrm{eV} \mathrm{cm}^{-3}$ and $U_{\mathrm{OPT}}=2.0 \mathrm{eV} \mathrm{cm}^{-3}$ are used to reproduce observed $\gamma$-ray SED. 